\title{
Coevolutionary dynamics of adaptive radiation for food-web development
}

\author{
Hiroshi C. Ito $\cdot$ Masakazu Shimada \\ Takashi Ikegami
}

Received: 10 April 2008/Accepted: 5 August 2008/Published online: 15 October 2008

(C) The Society of Population Ecology and Springer 2008

\begin{abstract}
To investigate how complex food-webs can develop through repeated evolutionary diversification, a predator-prey model was analyzed. In the model, each individual has two traits: trait $x$ as a predator and trait $y$ as a prey. These traits constitute a two-dimensional phenotype space, in which the whole group of individuals are represented as a phenotype distribution. Predator-prey interactions among the phenotypes are determined by their relative positions in the phenotype space. Each phenotypic cluster was treated as a species. Each species evolves in $y$ to escape from predation, while it evolves in $x$ to chase their prey. Analytical investigation provided two predictions. First, coupled evolutionary diversifications of $y$ and $x$ may occur when the $x$ of predators have caught up with their prey's $y$, which may be repeated. Second, complex food-webs may develop when species' competitive strengths are kept similar within the communities. If the functional response is close to the ratio-dependent response, the competitive strengths of all species are similar when the relationship between predators and prey corresponds to the ideal free distribution (IFD). These predictions were confirmed by numerical simulations.
\end{abstract}

Keywords Adaptive dynamics · Evolutionary branching · Ideal free distribution $\cdot$ Predator-prey $\cdot$ Speciation

Electronic supplementary material The online version of this article doi:(10.1007/s10144-008-0113-5) contains supplementary material, which is available to authorized users.

H. C. Ito $(\square) \cdot$ M. Shimada $\cdot$ T. Ikegami

The Graduate School of Arts and Sciences,

University of Tokyo, 3-8-1 Komaba, Meguro-ku,

Tokyo 153-8902, Japan

e-mail: itoh9@dolphin.c.u-tokyo.ac.jp

\section{Introduction}

The present biological communities being ultimately derived from a universal ancestor through repeated speciation is a widespread idea (Di Giulio 2001). The evolutionary history seems to have been influenced by both external and internal factors. Ecological interaction among organisms (e.g., predator-prey interaction and resource competition), which is one of the internal factors, induces their coevolution. Their evolutionary changes can also change the ways of interactions among them, which changes their ways of evolution further. Such a feedback process of coevolutionary dynamics may have induced the autonomous diversification and development of biological communities.

For the evolutionary development of a food-web, the number of its nodes has to increase at least. The number of nodes may increase with evolutionary branching of the component species through ecological interaction. Indeed, recent theoretical studies showed the possibility of evolutionary branching through various ecological interactions, including resource competition, predator-prey interaction, and mutualism (Geritz et al. 1998; Dieckmann and Doebeli 1999; Kisdi and Geritz 1999; Doebeli and Dieckmann 2000; Kisdi and Geritz 2001; Dercole 2003; Ackermann and Doebeli 2004; Egas et al. 2005; Kisdi 2006; Ito and Dieckmann 2007; Ito and Shimada 2007). If such situations occur repeatedly during the evolutionary dynamics, the community may develop from small and simple to large and complex.

Recently, several models for the evolutionary development of food-webs were developed (Caldarelli et al. 1998; Yoshida 2003; Drossel et al. 2004; Loeuille and Loreau 2005; Ito and Ikegami 2006). They differ from each other in their definition of the traits that determine predator-prey 
interaction. Especially, the number of traits ranges from one (Loeuille and Loreau 2005) to many (Caldarelli et al. 1998). Despite the difference, they have successfully demonstrated the coevolutionary formation of food-webs using numerical simulation. Ito and Ikegami (2006) has shown that foodwebs develop with repeated evolutionary branching.

In this paper, we will construct a similar food-web model to Ito and Ikegami (2006), assuming a two-dimensional phenotype space, and analytically investigating how evolutionary diversification can be repeated and how complex food-webs may develop. The obtained predictions are examined by numerical simulation.

\section{Model}

Main structure

Suppose there is a large and multi-dimensional phenotype space $\mathbf{s}$, in which most kinds of creatures can be expressed as phenotypes. Relationships among the phenotypes determine their predator-prey interactions. Assuming clonal reproduction, we write the biomass change of the $i$ th phenotype as:

$\frac{\mathrm{d} n_{i}}{\mathrm{~d} t}=\lambda \sum_{j}^{N} n_{i} \cdot g\left(\mathbf{s}_{j}, \mathbf{s}_{i}\right)-\sum_{j}^{N} n_{j} \cdot g\left(\mathbf{s}_{i}, \mathbf{s}_{j}\right)-\rho \cdot n_{i}$

where $N$ is the number of existing phenotypes, $g\left(\mathbf{s}_{j}, \mathbf{s}_{i}\right)$ denotes the predation rate by phenotype $i$ on $j$, i.e., functional response, constant $\lambda(<1)$ denotes the biomass production per unit predation amount, i.e., trophic efficiency, and constant $\rho$ denotes the rate of biomass loss by energy consumption and natural death.

\section{Functional response}

In real predator-prey interactions, functional responses depend on the relationship between the strategies of predators and their prey, both of which are important evolutionary components (Vermeij 1987). Following Ito and Ikegami (2006), we define the prey strategy of phenotype $\mathbf{s}$ as a resource distribution in a "resource space," $\mathbf{z}$, provided by a unit biomass of the phenotype, i.e., a "resource pattern": $r(\mathbf{z}, \mathbf{s})$. The resource space has arbitrary dimensions, and its axes may be body size, activity time, habitat, nutrient composition, hardness, toxicity, etc. The resource pattern can have a certain width because individuals with a same phenotype can have different resource properties among them, depending on their age, behavior, diet, environment, etc. On the other hand, we define the predator strategy of phenotype $\mathbf{s}$ as its energy investment distribution in the resource space provided by a unit biomass of the phenotype, i.e., a "consumption-effort distribution": $M \cdot c(\mathbf{z}, \mathbf{s})$. A constant $M$ is the total amount of consumption effort and $c(\mathbf{z}, \mathbf{s})$ determines its allocation to each resource, i.e., "consumption-effort pattern." The consumption-effort distribution is influenced by the size of the jaw or claw, performance of eyes, preference, digestion ability, etc.

The sum of the consumption-effort patterns of existing phenotypes gives the total consumption-effort distribution:

$C(\mathbf{z})=\sum_{i}^{N} n_{i} \cdot M \cdot c\left(\mathbf{z}, \mathbf{s}_{i}\right)$

while the total resource distribution, $R(\mathbf{z})$, is given by the sum of the resource patterns of existing phenotypes:

$R(\mathbf{z})=\sum_{i}^{N} n_{i} \cdot \Omega \cdot r\left(\mathbf{z}, \mathbf{s}_{i}\right)$

where the constant $\Omega$ denotes the detection rate of unit biomass of resource by a predator with unit searching effort. Thus $R(\mathbf{z})$ corresponds to visible resources for predators.

With $C(\mathbf{z})$ and $R(\mathbf{z})$, we define the functional response. For simplicity, we assume that the function of predation rate (on resource $\mathbf{z}$ per unit consumption effort) is independent of the predator's phenotype $\mathbf{s}$ and of the resource's property z. Following Ito and Shimada (2007), we express a functional response of phenotype $\mathbf{s}$ to resource $\mathbf{z}$ using a general form, known as the Beddington-DeAngelis type (Beddington 1975; DeAngelis et al. 1975):

$$
\begin{aligned}
g(\mathbf{z}, \mathbf{s}) & =\frac{M c(\mathbf{z}, \mathbf{s}) \cdot R(\mathbf{z})}{\alpha+\beta \cdot C(\mathbf{z})+\gamma \cdot R(\mathbf{z})} \\
& =M c(\mathbf{z}, \mathbf{s}) \phi(\mathbf{z}) \cdot R(\mathbf{z})
\end{aligned}
$$

where:

$$
\phi(\mathbf{z})=\frac{1}{\alpha+\beta \cdot C(\mathbf{z})+\gamma \cdot R(\mathbf{z})}
$$

where $\alpha, \beta$, and $\gamma$ are constant energy consumption rates, corresponding to the searching, interference competition, and the handling of resources, respectively. See S1 in the Electronic supplementary material (ESM) for the derivation of Eq. 4, which is a modification of Beddington (1975). Since $C(\mathbf{z})$ is the total consumption-effort on resource $\mathbf{z}$, it corresponds to the amount of competitors. When there is an abundance of resources with no competitors, Eq. 4 has its maximum value, $\operatorname{Mc}(\mathbf{z}, \mathbf{s}) / \gamma$. The increase of competitors $C(\mathbf{z})$ always reduces the response. Note that this becomes the type II response (Holling 1959) or the ratio-dependent response (Arditi and Ginzburg 1989) when $\beta=0$ or $\alpha=0$, respectively.

Then, the functional response of a unit biomass of $\mathbf{s}_{i}$ to resource $\mathbf{z}$ is given by $g\left(\mathbf{z}, \mathbf{s}_{i}\right)$, and the fraction of $\mathbf{s}_{j}$ in the resource is given by $n_{j} \Omega r\left(\mathbf{z}, \mathbf{s}_{j}\right) / R(\mathbf{z})$. Thus, the functional response of $\mathbf{s}_{i}$ to $\mathbf{s}_{j}$ is obtained as: 


$$
\begin{aligned}
g\left(\mathbf{s}_{j}, \mathbf{s}_{i}\right) & =\int g\left(\mathbf{z}, \mathbf{s}_{i}\right) \cdot \frac{n_{j} \Omega r\left(\mathbf{z}, \mathbf{s}_{j}\right)}{R(\mathbf{z})} \mathrm{d} \mathbf{z} \\
& =M \Omega n_{j} \int \phi(\mathbf{z}) c\left(\mathbf{z}, \mathbf{s}_{i}\right) r\left(\mathbf{z}, \mathbf{s}_{j}\right) \mathrm{d} \mathbf{z}
\end{aligned}
$$

where $\mathrm{d} \mathbf{z}$ denotes the volume element of the resource space. Substituting this into Eq. 1 yields:

$$
\begin{aligned}
& \frac{\mathrm{d} n_{i}}{\mathrm{~d} t}=\lambda \sum_{j}^{N} a_{j i} \cdot n_{i} n_{j}-\sum_{j}^{N} a_{i j} \cdot n_{j} n_{i}-\rho \cdot n_{i} \\
& a_{i j}=M \Omega \int \phi(\mathbf{z}) c\left(\mathbf{z}, \mathbf{s}_{i}\right) r\left(\mathbf{z}, \mathbf{s}_{j}\right) \mathrm{d} \mathbf{z}
\end{aligned}
$$

Here, $a_{j i}$ indicates the strength of the interaction between phenotypes $\mathbf{s}_{i}$ (as the predator) and $\mathbf{s}_{j}$ (as the prey), which corresponds to the $(i, j)$ element of the community matrix of the system. If no saturation of predation and no interference competition are assumed (i.e., $\beta=\gamma=0$ ), the functional response corresponds to type $\mathrm{I}$, and $\alpha_{i j}$ is determined only with $\mathbf{s}_{i}$ and $\mathbf{s}_{j}$. In this case, Eq. 7 becomes the standard Lotka-Volterra predator-prey equation. Otherwise, Eq. 4 does not allow such simplification. To make the form of Eq. 7 simpler, we transform it into:

$$
\begin{aligned}
\frac{1}{n_{i}} \cdot \frac{\mathrm{d} n_{i}}{\mathrm{~d} t}= & \lambda M \int c\left(\mathbf{z}, \mathbf{s}_{i}\right) \phi(\mathbf{z}) R(\mathbf{z}) \mathrm{d} \mathbf{z} \\
& -\Omega \int r\left(\mathbf{z}, \mathbf{s}_{i}\right) \phi(\mathbf{z}) C(\mathbf{z}) \mathrm{d} \mathbf{z}-\rho
\end{aligned}
$$

In this expression, each phenotype $\mathbf{s}_{i}$ interacts as a predator with the total resource distribution $R(\mathbf{z})$ and interacts as prey with the total consumption-effort distribution $C(\mathbf{z})$.

The model is still incomplete, since it does not have any resource inflow, in which the system cannot sustain its total biomass. Here, we add a basal resource, $I(\mathbf{z})$, to the total resource distribution:

$R(\mathbf{z})=\sum_{i}^{N} n_{i} \cdot \Omega \cdot r\left(\mathbf{z}, \mathbf{s}_{i}\right)+\Omega \cdot I(\mathbf{z})$

The functional response of phenotype $i$ to $I(\mathbf{z})$ is given by:

$$
\int g\left(\mathbf{z}, \mathbf{s}_{i}\right) \cdot \frac{I(\mathbf{z})}{R(\mathbf{z})} \mathrm{d} \mathbf{z}=M \Omega \int c\left(\mathbf{z}, \mathbf{s}_{i}\right) \phi(\mathbf{z}) I(\mathbf{z}) \mathrm{d} \mathbf{z}
$$

in the same manner as with Eq. 6. Thus, the dynamics of $I(\mathbf{z})$ can be written as:

$$
\begin{aligned}
\frac{\mathrm{d} I}{\mathrm{~d} t} & =v\left(I_{0}-I\right)-\sum_{j}^{N}\left[n_{j} \cdot M \Omega \int c\left(\mathbf{z}, \mathbf{s}_{j}\right) \phi(\mathbf{z}) I(\mathbf{z}) \mathrm{d} \mathbf{z}\right], \\
& =v\left(I_{0}-I\right)-\Omega \int I \cdot r_{I}(\mathbf{z}) \phi(\mathbf{z}) C(\mathbf{z}) \mathrm{d} \mathbf{z}
\end{aligned}
$$

where $I$ is the amount of basal resource and $r_{I}(\mathbf{z})$ is its resource pattern. A constant $v$ determines the resource inflow and outflow (or decay), which are given by $v I_{0}$ and $v I$, respectively.
With arbitrary definitions of $\mathbf{s}, \mathbf{z}, c(\mathbf{z}, \mathbf{s}), r(\mathbf{z}, \mathbf{s})$, and $I(\mathbf{z})$, the population dynamics and evolutionary dynamics can be analyzed using Eqs. 8 and 11. In this study, for simplicity, we assume a one-dimensional resource space, $\mathbf{z}=z$. In addition, we assume a two-dimensional phenotype space $\mathbf{s}=(x, y)$, in which traits $x$ and $y$ have real numbers and affect only the positions of $c(z, \mathbf{s})$ and $r(z, \mathbf{s})$, respectively. In this case, $c(z, \mathbf{s})$ and $r(z, \mathbf{s})$ are written as:

$$
\begin{aligned}
& c(z, \mathbf{s})=c(z-x) \\
& r(z, \mathbf{s})=r(z-y)
\end{aligned}
$$

Under this assumption, phenotype $\mathbf{s}_{i}$ consumes resources around $z=x_{i}$, while it behaves as resources around $z=y_{i}$, which may be consumed by other phenotypes (Fig. 1a). A phenotype whose $x$ is similar to its $y$ preys on itself (i.e., cannibalism). If $x$ and $y$ are gravity centers of the consumption-effort and resource patterns, respectively, $x$ and $y$ may be taken as genetic values in quantitative genetics. In this case, the widths of those patterns correspond to environmental variances.

Analysis of coevolutionary dynamics

To make the analysis simple, we assume that the consumption-effort and resource patterns share the same distribution function $h(z)$ :

$c(z, \mathbf{s})=h(z-x)$

$r(z, \mathbf{s})=h(z-y)$

Here, $h(z)$ is a unimodal and symmetrical function (i.e., $h(-z)=h(z)$ ), with a single maximum at $z=0$ (Fig. 1b). The function is nonnegative and smooth within its range, and its integration gives unity. $h(z)$ can be a Gaussian distribution. We also assume that the resource pattern of the basal resource also shares the $h(z)$ distribution, i.e., $r_{I}(z)$ $=h\left(z-z_{L}\right)$. In addition, we assume very fast resource updating by choosing $v$ to be very large compared to its consumption rate by existing phenotypes, at Eq. 11. In this case, it has a constant distribution, $I(z)=I_{0} h\left(z-z_{L}\right)$. Such a basal resource may be taken as the sunlight.

\section{Directional coevolution}

We analyze the evolutionary dynamics based on the adaptive dynamics theory sensu stricto (Dieckmann and Law 1996; Metz et al. 1996; Geritz et al. 1997). A very small mutation rate is assumed so that the system is always at population dynamical equilibrium when a mutant has emerged. Here, we assume that the values of the model parameters are appropriately chosen so that the system has a stable equilibrium in many cases. At such an equilibrium, increasing rates of existing phenotypes are equal to zero, and each population has only a single phenotype in most 
(a)

Phenotype space: $s=(x, y)$

$x:$ Trait as predator (consumer)

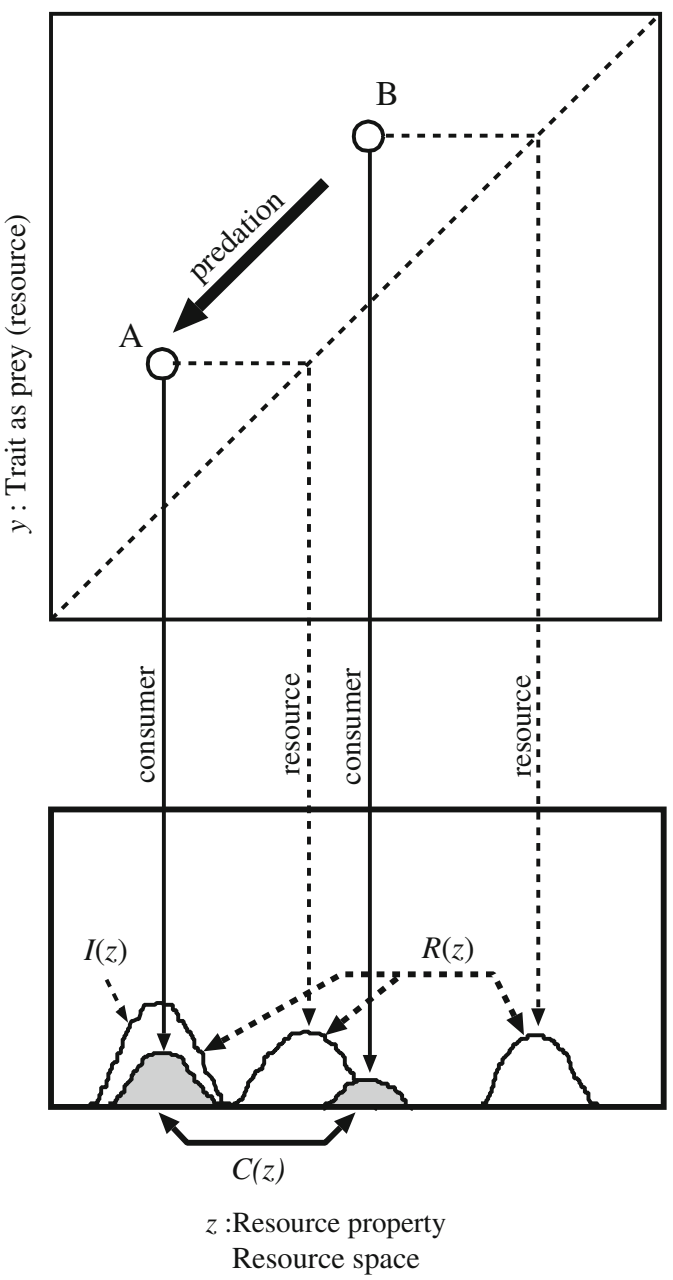

(b)

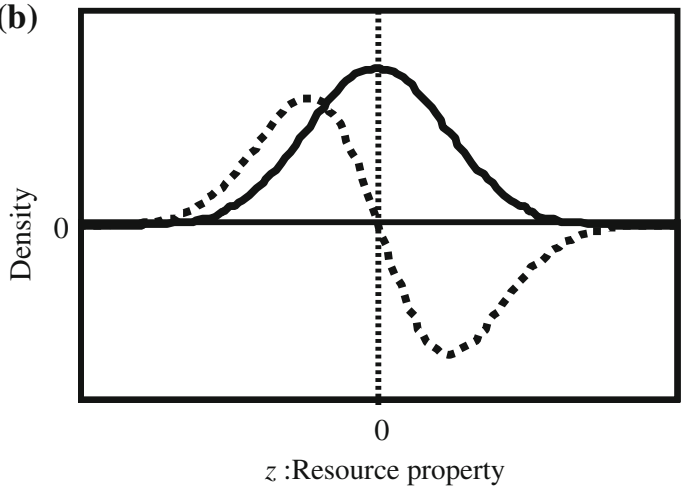

Fig. 1 The phenotype space, resource space, and predator-prey relationship. a A predator-prey relationship between two phenotypes is represented. The diagonal dotted line indicates $x=y$. The solid arrows from phenotypes $A$ and $B$ indicate their consumption effort, while the dotted arrows indicate locations where they exist as resources. $\mathbf{b}$ The shape of the distribution function, $h(z)$, which is used for the consumption-effort and resource patterns in analytical investigation, is shown as the solid curve and its first derivative (i.e., its gradient) is shown as the dashed curve cases. We call such populations separated from each other "species" for convenience. Suppose a mutant $\mathbf{s}^{\prime}=\left(x^{\prime}, y^{\prime}\right)$ emerged in species $i$. The deviation of $\mathbf{s}^{\prime}$ from its parent phenotype $\mathbf{s}_{i}$ is assumed to be slight. We denote its biomass using $n^{\prime}$. Its invasion fitness, which is its initial per-capita growth rate, is given by Eq. 8, with $n^{\prime} \rightarrow 0$ :

$$
\begin{aligned}
f\left(\boldsymbol{s}^{\prime} ; \boldsymbol{S}, \hat{\boldsymbol{n}}\right)= & \lim _{n^{\prime} \rightarrow 0}\left[\frac{1}{n^{\prime}} \cdot \frac{\mathrm{d} n^{\prime}}{\mathrm{d} t}\right]=\lambda M \int c\left(z, \boldsymbol{s}^{\prime}\right) \phi(z) R(z) \mathrm{d} z \\
& -\Omega \int r\left(z, \boldsymbol{s}^{\prime}\right) \phi(z) C(z) \mathrm{d} z-\rho .
\end{aligned}
$$

Here, $\boldsymbol{S}$ denotes a vector of phenotypes of existing species, $\boldsymbol{S}=\left(\boldsymbol{s}_{1}, \ldots, \boldsymbol{s}_{N}\right)$, and $\hat{\boldsymbol{n}}=\left(\hat{n}_{1}, \ldots, \hat{n}_{N}\right)$ denotes their equilibrium biomasses. These biomasses are used for the calculation of $R(z), C(z)$, and $\phi(z)$ according to Eqs. 2, 3, and 5. (The $\hat{\boldsymbol{n}}$ is included as the argument of the invasion fitness, since one $\boldsymbol{S}$ might have more than one equilibrium.) If the mutant has a positive invasion fitness, it can invade the community, which usually replaces its parent phenotype $\mathbf{s}_{i}$ and coexists with other species. Repetition of this process corresponds to directional evolution.

Computing the invasion fitnesses of various $\boldsymbol{s}^{\prime}$, a fitness landscape around $\mathbf{s}_{i}$ can be generated. The strengths of directional selections on species $i$ in $x$ and $y$ are defined by first derivatives of the fitness landscape at $\boldsymbol{s}^{\prime}=\boldsymbol{s}_{i}$ :

$$
\begin{aligned}
G_{x}\left(\mathbf{s}_{i}\right) & =\left[\frac{\partial f\left(\boldsymbol{s}^{\prime} ; \boldsymbol{S}, \hat{\boldsymbol{n}}\right)}{\partial x^{\prime}}\right]_{\mathbf{s}^{\prime}=\mathbf{s}_{i}} \\
& =\lambda M \frac{\partial}{\partial x^{\prime}}\left[\int c\left(z, \boldsymbol{s}^{\prime}\right) \cdot \phi(z) R(z) \mathrm{d} z\right]_{\mathbf{s}^{\prime}=\mathbf{s}_{i}} \\
G_{y}\left(\mathbf{s}_{i}\right) & =\left[\frac{\partial f\left(\boldsymbol{s}^{\prime} ; \boldsymbol{S}, \hat{\boldsymbol{n}}\right)}{\partial y^{\prime}}\right]_{\mathbf{s}^{\prime}=\mathbf{s}_{i}} \\
& =-\Omega \frac{\partial}{\partial y^{\prime}}\left[\int r\left(z, \mathbf{s}^{\prime}\right) \cdot \phi(z) C(z) \mathrm{d} z\right]_{\mathbf{s}^{\prime}=\mathbf{s}_{i}}
\end{aligned}
$$

respectively. These form a selection gradient, $\left(G_{x}\left(\boldsymbol{s}_{i}\right)\right.$, $\left.G_{y}\left(s_{i}\right)\right)$, at $s_{i}$ in the phenotype space. The direction of the directional evolution is determined by the selection gradient. The $\int c\left(z, \boldsymbol{s}^{\prime}\right) \phi(z) R(z) \mathrm{d} z$ in Eq. 17 and $\int r\left(z, \mathbf{s}^{\prime}\right) \phi(z) C(z) \mathrm{d} z$ in Eq. 18 correspond to the biomass gain (as a predator) and loss (as prey) of $\boldsymbol{s}^{\prime}$, according to Eq. 8. Here, we call them the "resource gain of $s^{\prime \prime}$ " and the "predation pressure on $\boldsymbol{s}^{\prime}$ ", respectively. Because $\int c\left(z, \boldsymbol{s}^{\prime}\right) \mathrm{d} z=1$, the resource gain is a weighted average of $\phi(z) R(z)$ around $c\left(z, s^{\prime}\right)$. Then, we call $\phi(z) R(z)$ the "resource gain density at $z$." Similarly, the predation pressure is a weighted average of $\phi(z) C(z)$ around $r\left(z, s^{\prime}\right)$. We call $\phi(z) C(z)$ the "predation pressure density at $z$."

Equation 17 indicates that the position of the consumption-effort pattern, $x_{i}$, evolves toward the higher resource gain density in the resource space, if there exists a gradient of the resource gain density around $z=x_{i}$. 
Similarly, the position of the resource pattern, $r_{i}$, evolves toward the lower predation pressure density, according to Eq. 18 (see S2 in the ESM for details).

In the numerical simulation, the directional coevolution is composed of evolutionary shifts of several clumps of interacting predators and prey in the resource space (Fig. 5). Here, we call such clumps, "interaction groups," and suppose that they are isolated from each other. Then, in each interaction group, the predators (i.e., species whose $x$ belong to this group) evolve their $x$ to catch up with their prey (i.e., species whose $y$ belong to this group), while the prey evolve their $y$ to escape from the predation, as illustrated in Fig. 2a, d, h. Consequently, the $x$ of the predators and the $y$ of the prey evolve in the same direction in the resource space.

\section{Coevolutionary diversification}

Next, we analyze how evolutionary diversification including evolutionary branching may occur. To make the logic simple, we focus on a single interaction group that has only one predator species and one prey species. We assume that the group is well isolated from the others, as illustrated in Fig. 2a. We denote the prey and predator species with $i 0$ and $j 0$, respectively. Directional coevolution of the interaction group stops when the predator's $x$ has caught up with the prey's $y$, which means that $x_{j 0}=y_{i 0}$ (Fig. 2b). In this case, the directional selection on $x_{j 0}$ as well as that on $y_{i 0}$ has vanished (i.e., $x_{j 0}=y_{i 0}$ gives $G_{x}\left(\boldsymbol{s}_{j 0}\right)=G_{y}\left(\boldsymbol{s}_{i 0}\right)=0$ ). Then, the second derivatives of the invasion fitness, which are curvatures of the fitness landscape, become important determinants of the subsequent dynamics. They are defined by:

$$
\begin{aligned}
D_{y y}\left(\boldsymbol{s}_{i 0}\right) & =\left[\frac{\partial^{2} f\left(\boldsymbol{s}^{\prime} ; \boldsymbol{S}, \hat{\boldsymbol{n}}\right)}{\partial y^{\prime 2}}\right]_{\mathbf{s}^{\prime}=\mathbf{s}_{i 0}} \\
& =-\Omega \frac{\partial^{2}}{\partial y^{\prime 2}}\left[\int r\left(z, \boldsymbol{s}^{\prime}\right) \cdot \phi(z) C(z) \mathrm{d} z\right]_{\mathbf{s}^{\prime}=\mathbf{s}_{i 0}} \\
D_{x x}\left(\boldsymbol{s}_{j 0}\right) & =\left[\frac{\partial^{2} f\left(\boldsymbol{s}^{\prime} ; \boldsymbol{S}, \hat{\boldsymbol{n}}\right)}{\partial x^{\prime 2}}\right]_{\mathbf{s}^{\prime}=\mathbf{s}_{j 0}} \\
& =\lambda M \frac{\partial^{2}}{\partial x^{\prime 2}}\left[\int c\left(z, \boldsymbol{s}^{\prime}\right) \cdot \phi(z) R(z) \mathrm{d} z\right]_{\mathbf{s}^{\prime}=\mathbf{s}_{j 0}}
\end{aligned}
$$

If $D_{y y}\left(s_{i 0}\right)$ is positive, the fitness landscape forms a valley at $\boldsymbol{s}_{i 0}$ in the direction of $y$, which may induce diversification of the prey in $y$. Similarly, if $D_{x x}\left(\boldsymbol{s}_{j 0}\right)$ is positive, it may induce diversification of the predator in $x$. With the consumptioneffort and resource patterns defined in Eqs. 14 and 15, $D_{y y}\left(\boldsymbol{s}_{i 0}\right)>0$ and $D_{x x}\left(\boldsymbol{s}_{j 0}\right)<0$ are always held under any biomasses for the predator and the prey if $h(z)$ has no more than two inflection points at least, as in the case of a Gaussian distribution (S3 in the ESM). Thus, evolutionary branching of the prey's $y$ may occur (Fig. 2b, c). When this has occurred, diversified prey form a bimodal resource distribution for the predator (Fig. 2c), which may induce diversifying selection in the predator's $x$ and favor its evolutionary branching (Fig. 2c, d), as in Doebeli and Dieckmann (2000). If an interaction group has more than one predator and/or prey species, trait substitution of the prey and/or the predators may occur instead of evolutionary branching (Fig. 2f-h). Here, we call such evolutionary diversification of the resource pattern, including evolutionary branching and trait substitution, " $r$-diversification," while we call those of the consumption-effort pattern, " $c$ diversification." The coupled $r$ - and $c$-diversifications correspond to the division of an interaction group, which results in directional coevolution of the divided interaction groups again (resource spaces in Fig. 2a-d and f-h). If predators catch up with their prey after each of the such coupled diversifications, the diversifications can occur repeatedly (Fig. 2e), which effectively increases the number of species.

\section{Maintenance of complex food-webs}

Complex food-webs should have interaction groups with several prey and/or several predators (otherwise, only food chains with no branches are possible). Such an interaction group is generated by the evolutionary branching of its component species or the fusion of interaction groups. The fusion occurs by the collision of interaction groups in the resource space. Here, we examine how the coexistence of several prey and predators can be maintained in an interaction group in the time scale of food-web development (i.e., during a time period in which the system has several divisions and fusions of interaction groups at least). Suppose we have an interaction group with two predator species and two prey species, as illustrated in Fig. 3b. The population dynamics of predator species $j 1$ and $j 2$ are given by:

$$
\begin{aligned}
\frac{1}{n_{j 1}} \cdot \frac{\mathrm{d} n_{j 1}}{\mathrm{~d} t}= & \lambda M \int c\left(z, s_{j 1}\right) \phi(z) R(z) \mathrm{d} z \\
& -\Omega \int r\left(z, s_{j 1}\right) \phi(z) C(z) \mathrm{d} z-\rho \\
\frac{1}{n_{j 2}} \cdot \frac{\mathrm{d} n_{j 2}}{\mathrm{~d} t}= & \lambda M \int c\left(z, s_{j 2}\right) \phi(z) R(z) \mathrm{d} z \\
& -\Omega \int r\left(z, s_{j 2}\right) \phi(z) C(z) \mathrm{d} z-\rho
\end{aligned}
$$

Since $j 1$ and $j 2$ belong to the same interaction group, $x_{j 1}$ and $x_{j 2}$ are close to each other. This makes their resource gains (i.e., the first terms on the right sides of the above equations) always similar, although the magnitude relation of the gains can change depending on $n_{j 1}$ and $n_{j 2}$.

If there is a large difference in predation pressure by their predators at higher trophic levels (e.g., the second terms in the above equations), it cannot be absorbed by the 
(a)

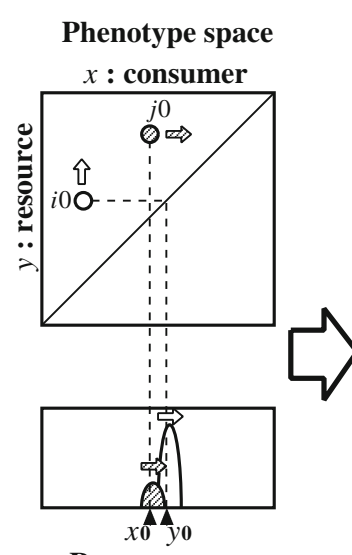

Resource space

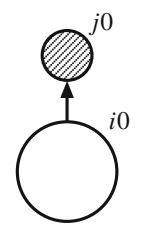

(f)

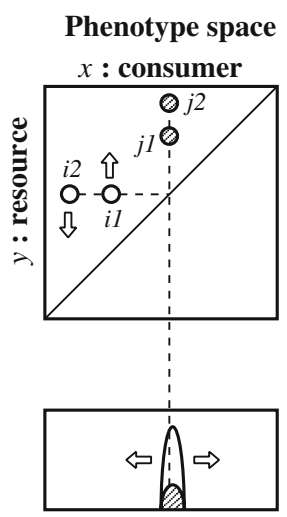

Resource space

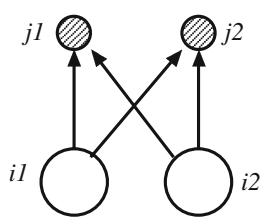

(b)

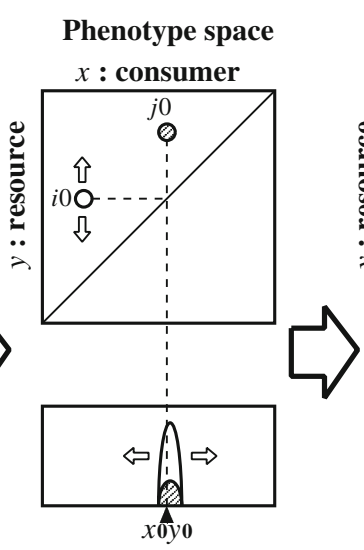

Resource space

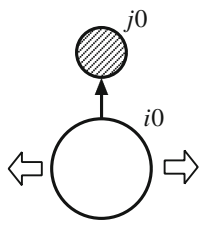

(g)

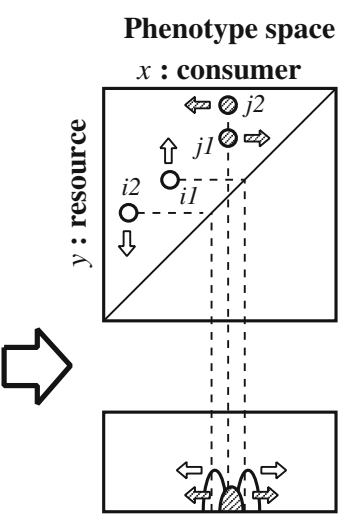

Resource space

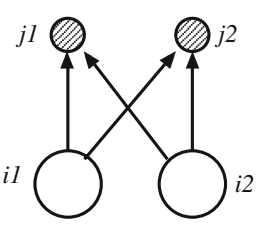

(c)

Phenotype space $x$ : consumer

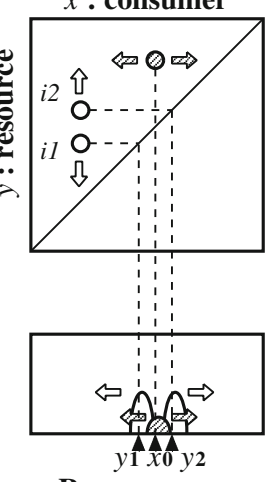

Resource space

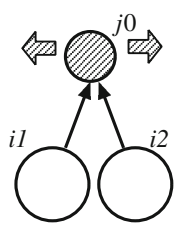

(h) (d)

Phenotype space

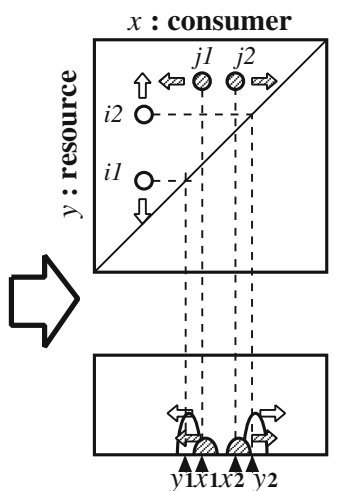

Resource space

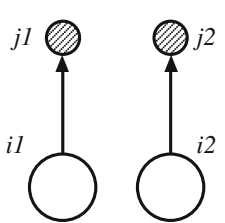

(e)

Phenotype space
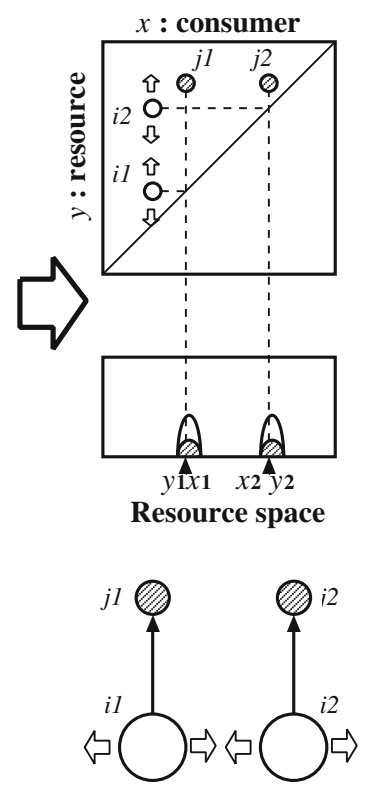

Fig. 2 Directional coevolution and coupled $r$-and $c$-diversifications of interaction groups. In each panel, the local phenotype distribution, corresponding interaction groups in the resource space, and corresponding local food-web are illustrated. Species are indicated with small circles in the phenotype space. The dotted lines from species indicate the locations of their consumption-effort patterns (vertical

small difference of their resource gains. For example, suppose species $j 1$ experiences a much lower predation pressure than $j 2$ (e.g., $\int r\left(z, \boldsymbol{s}_{j 1}\right) \phi(z) C(z) \mathrm{d} z<\int r\left(z, \boldsymbol{s}_{j 2}\right) \phi$ $(z) C(z) \mathrm{d} z)$. In this case, $j 1$ is competitively much stronger than $j 2$, because it is tolerable against a much lower resource gain. Thus, $j 1$ exploits the resource around the
Phenotype space

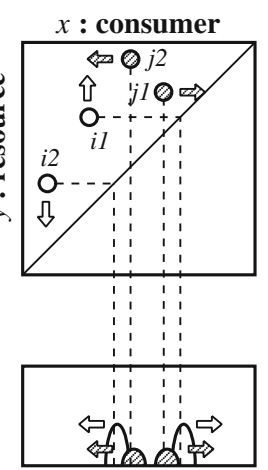

Resource space

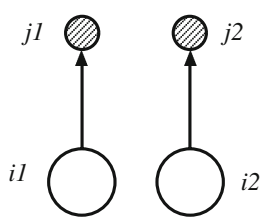

straight lines) or their resource patterns (lines reflected at the diagonal lines) in the resource space. In the resource space, the shaded and unshaded distributions indicate the total consumptioneffort and the total resource distributions, respectively. The arrows in the phenotype and resource spaces indicate the directions of their evolutionary branching or directional evolution

consumption-effort patterns of $j 1$ and $j 2$, such that $j 2$ is excluded by too low a resource density $\phi(z) R(z)$ for it. Even if $x_{j 1}$ and $x_{j 2}$ are different so that $j 2$ can still survive, the resource consumed by $j 2$ has a much higher density than that consumed by $j 2$, like an empty niche for the stronger $j 2$. This induces rapid directional evolution or 
(a)

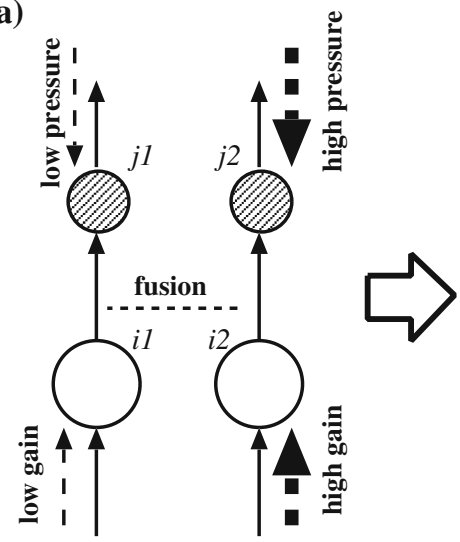

(c)
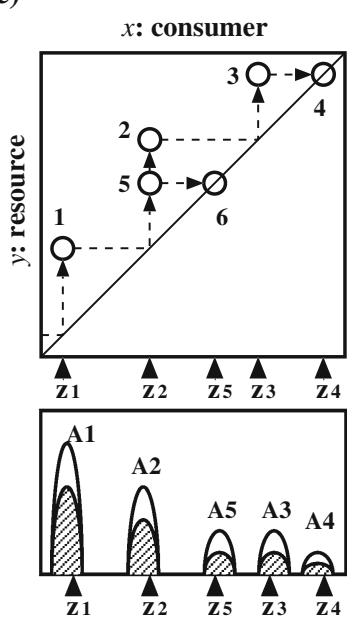

Resource space (b)

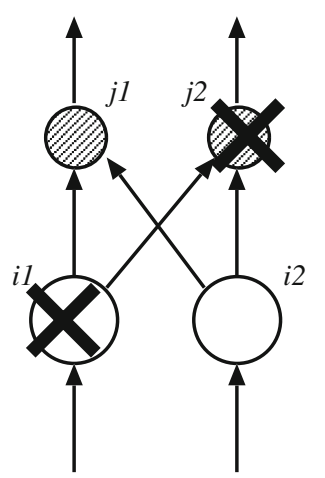

(d)

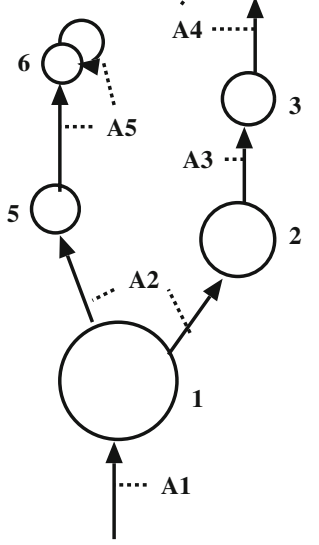

Fig. 3 Extinction by fusion. Possible extinction by the fusion of two interaction groups is illustrated in $\mathbf{a}$ and $\mathbf{b}$. $\mathbf{c}$ An example of a no-lag community, which may avoid such an extinction. Its food-web structure is illustrated in $\mathbf{d}$

evolutionary diversification of $x_{j 1}$ toward $x_{j 2}$, excluding $j 2$ (Ito and Dieckmann 2007). Thus, the predation pressures on $j 1$ and $j 2$ have to be similar in order to allow their coexistence in the time scale of food-web development. Note that this logic is essentially the same with the general concept of the "limiting similarity" (MacArthur 1972).

As for the prey species $i 1$ and $i 2$, if there exists a large difference in their resource gains (e.g., $\int c\left(z, s_{i 1}\right) \phi(z) R(z)$ $\left.\mathrm{d} z<\int c\left(z, \boldsymbol{s}_{i 2}\right) \phi(z) R(z) \mathrm{d} z\right)$, the competitively stronger $i 2$ with larger resource gain is likely to exclude $i 1$ through apparent competition under the shared predation pressures by species $j 1$ and $j 2$.

In general, the coexistence of several predator species in an interaction group requires that the predation pressures on them (i.e., competitive strength as the predator) are similar, while the coexistence of several prey species requires that their resource gains (i.e., competitive strength as the prey) are similar. In addition, the similarity in the competitive strength between interaction groups are also required to avoid extinction by their fusion (Fig. 3a, b), with the same logic. If the resource gain density $\phi(z) R(z)$ and the predation pressure density $\phi(z) C(z)$ are flat in the resource space, the competitive strengths as both predator and as prey are identical among all existing species. This is realized if the functional response is close to the ratiodependent response (i.e., small $\alpha$ at Eq. 4), and if $C(z)$ is close to the ideal free distribution (IFD) (Fretwell and Lucas 1970) in the evolutionary sense, as explained below.

An IFD is a distribution of consumers among resources so that every consumed resource has an identical quality (Fretwell and Lucas 1970). In our model, an evolutionary IFD (E-IFD) can be defined as a consumption distribution $C(z)$ that makes the resource gain density $\phi(z) R(z)$ flat in the resource space, in which absent resources (i.e., $z$ with $R(z)=0$ ) are neglected. While the original concept of IFD assumes extremely efficient foraging behaviors of predators compared to the escaping behaviors of prey, the state considered here requires extremely fast evolution of consumption-effort patterns compared to that of resource patterns. Under $\alpha=0$ in Eq. 4 , in which the response is ratio-dependent, $\phi(z) R(z)$ and the predation pressure density $\phi(z) C(z)$ can be transformed as:

$$
\begin{aligned}
& \phi(z) R(z)=\frac{1}{\beta A(z)+\gamma} \\
& \phi(z) C(z)=\frac{A(z)}{\beta A(z)+\gamma}=\frac{1}{\beta}-\frac{\gamma}{\beta} \cdot \phi(z) R(z)
\end{aligned}
$$

where $A(z)=C(z) / R(z)$. Thus, flat $\phi(z) R(z)$ gives flat $A(z)$, which gives flat $\phi(z) C(z)$. Since the system is almost always at population dynamical equilibrium, the value of $A(z)$ is determined by the condition for population dynamical equilibrium from Eq. 8, that is:

$A(z)=\frac{C(z)}{R(z)}=\frac{\lambda M-\gamma \rho}{\Omega+\beta \rho}=\hat{A}$

where $\lambda M-\gamma \rho>0$ should be fulfilled, otherwise, any phenotype cannot keep its biomass. An E-IFD is realized if $x$ of every species has caught up with its prey's $y$ (Fig. 3c). In this case, there is no evolutionary lag between predators and prey in every interaction group. We call such a set of existing phenotypes a "no-lag community" and denote it with $\hat{\boldsymbol{S}}=$ $\left(\hat{\boldsymbol{s}}_{1}, \ldots, \hat{\boldsymbol{s}}_{N}\right)$. In a no-lag community, for any $i=1, \ldots, N$, the equal value to $y_{i}$ can be found among $x_{j}(j=1, \ldots, N)$, and vice versa. Any no-lag community can have E-IFD through population dynamics and it gives an equilibrium. Many of such equilibria seem to be locally stable in terms of the dynamics of $C(z)$ and $R(z)$ (numerically examined) (see $\mathrm{S} 4$ in the ESM). Thus, it is expected that $C(z)$ can be kept close to E-IFD if the evolution of $x$ is sufficiently fast compared to $y$.

Similar logic to that above holds even if shapes and their mutational changes of the consumption-effort and resource 
patterns are differently defined from Eqs. 14 and 15 (e.g., mutation in their widths), as explained below. Consumption-effort patterns evolve toward higher resource gain densities $\phi(z) R(z)$ from lower ones in the resource space. Since $\phi(z) R(z)$ is a monotonically decreasing function of $A(z)=C(z) / R(z)$, it can be said that consumption-effort patterns evolve toward smaller $C(z) / R(z)$. Such evolution of those patterns changes $C(z)$ so that large $C(z) / R(z)$ becomes smaller, while small $C(z) / R(z)$ becomes larger. Thus, the evolutionary change of $C(z)$ makes $C(z) / R(z)$ flatter, which corresponds to a smaller deviation of $C(z)$ from E-IFD, $\hat{A} R(z)$. In other words, the evolutionary change of $C(z)$ makes itself more similar to $R(z)$.

On the other hand, resource patterns evolve toward lower predation pressure densities $\phi(z) C(z)$ from higher ones in the resource space. Since $\phi(z) C(z)$ is a monotonically increasing function of $A(z)=C(z) / R(z)$, it can be said that resource patterns evolve toward smaller $C(z) / R(z)$. Such evolution of those patterns changes $R(z)$ so that small $C(z) / R(z)$ becomes smaller, while large $C(z) / R(z)$ becomes larger. Thus, the evolutionary change of $R(z)$ increase the variation of $C(z) / R(z)$, which corresponds to larger deviations of $C(z)$ from E-IFD, $\hat{A} R(z)$. In other words, the evolutionary change of $R(z)$ makes itself more dissimilar to $C(z)$.

Thus, when the evolution of consumption-effort patterns is sufficiently faster than that of resource patterns, the deviation of $C(z)$ from E-IFD can be kept small, in which competitive strengths among species are kept similar. Conversely, if the evolutionary change of $C(z)$ is slow, a large deviation is expected. In this sense, the deviation of $C(z)$ from E-IFD at the population dynamical equilibrium may be treated as a general measure for evolutionary lag between predators and prey in the system. Thus, we measure the lag as an average of the local deviations of $C(z) / \hat{A} R(z)$ from 1 in the resource space:

$L=\frac{1}{V} \int\left[\log \left(\frac{C(z)+\varepsilon_{L}}{\hat{A} R(z)+\varepsilon_{L}}\right)\right]^{2} \mathrm{~d} z$

where $V$ is the size of the resource space, given by $\int \mathrm{d} z$, and a small constant $\varepsilon_{L}$ is introduced to keep the logarithm finite at $C(z)=0$ and/or $R(z)=0$. In the case of no-lag communities, which hold $C(z) / \hat{A} R(z)=1, L=0$ is fulfilled.

Even if $\alpha>0, C(z) / \hat{A} R(z) \simeq 1$ is fulfilled at E-IFD (i.e., flat $\phi(z) R(z))$ under sufficiently small $\alpha$ such that $\alpha \ll$ $\beta C(z)+\gamma R(z)$ holds. Then, $\phi(z) C(z)$ is also approximately flat. In this case, the competitive strengths are similar among extant species. It is also expected that population dynamical equilibria fulfilling $C(z) /[\hat{A} R(z)]=1$ is attained by sets of phenotypes that are similar to no-lag communities. In addition, the index $L$ still works as a measure of the deviation from E-IFD in this case.
In summary, under sufficiently small $\alpha$, food-web structures may be maintained easily if the evolutionary lag between predators and prey in the system (i.e., the deviation of $C(z)$ from E-IFD) is kept small by keeping the set of existing phenotypes similar to no-lag communities.

Potential evolutionary paths for the development of complex food-webs

The next question is how complex food-webs can develop from simple ones, under the constraint of small evolutionary lag. To examine this in a simple manner, we consider the case that the lag is infinitesimally small, which corresponds to no-lag communities. In this case, the $y$ of the prey and $x$ of the predators in the same interaction group are almost the same. The $C(z) /[\hat{A} R(z)]=1$ always gives population dynamical equilibrium through such changes, under $\alpha=0$ (see S4 in the ESM).

It is graphically shown that such changes can represent various topological transitions of food-webs (Fig. 2 assuming no lag and Fig. 4). The division of interaction groups (i.e., the coupled $r$ - and $c$-diversifications) can generate new species at existing trophic levels (Fig. 2) or at new trophic levels (Fig. 4a). On the other hand, fusions of interaction groups can generate omnivory and loops (Fig. 4b, c). There seems to be many other ways of such continuous changes of the phenotype distribution, which represent various topological transitions of food-webs. By combining them as transition sequences through continuous changes of the phenotype distribution, various potential paths for the development of complex food-webs from a single species are provided. Thus, if selection pressures induce evolutionary dynamics that are close to some of those potential paths, a complex food-web might develop.

However, a no-lag community does not have directional selection (but has stabilizing or diversifying selection) in any species, according to Eqs. 17 and 18. Since evolutionary branching requires the process of directional evolution to lead polymorphism into distinct two populations (Metz et al. 1996; Geritz et al. 1997), the number of species may not increase easily if the system is kept too close to no-lag communities. In this case, the development of complex food-webs might be difficult.

Summary of analytical results

The above analyses provide two predictions. First, the coupled $r$-and $c$-diversifications may occur when the $x$ of predators have caught up with their prey's $y$, which may be repeated. Second, complex food-webs might develop easily when the evolutionary lag between predators and prey in the system (i.e., the deviation of $C(z)$ from E-IFD) is kept small but not too small, if the functional response is close 
(a)
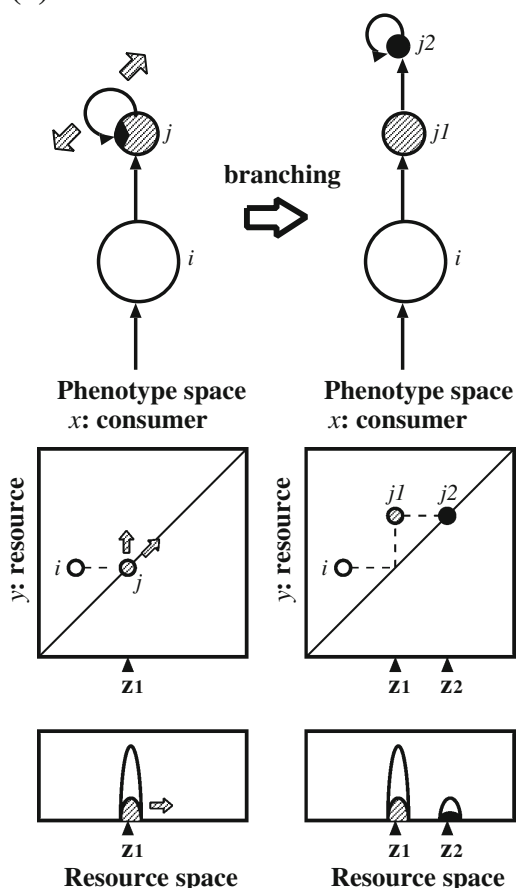

(b)

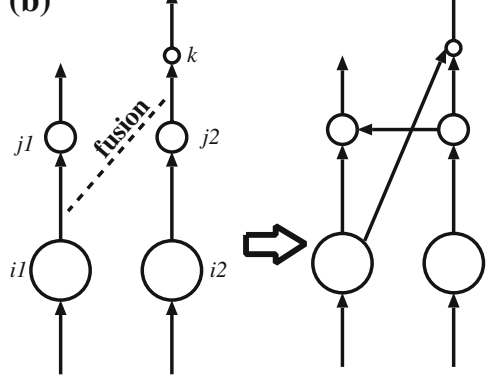

Phenotype space $x$ : consumer
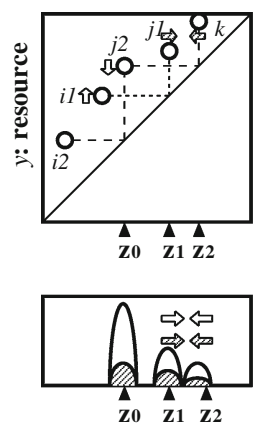

Resource space $x$ : consumer

Fig. 4 Examples of divisions and fusions of interaction groups without evolutionary lag between predators and prey. a An example of the emergence of a new trophic level. $\mathbf{b}$ and $\mathbf{c}$ Examples of the

to the ratio-dependent response. These predictions hold true, even if the phenotype and resource spaces have higher dimensionalities. The second prediction holds for arbitrary shapes and dynamics of the basal resource $I(z)$ if the system can have stable population dynamical equilibrium during the evolutionary dynamics. In this case, the first prediction also holds if species consuming the basal resource are treated as basal resources instead. In addition, the second prediction holds true for arbitrary shapes and mutational changes of consumption-effort and resource patterns.

Note that we do not know the stability of the population dynamical equilibria of communities that are close to nolag communities. In addition, we do not know how the likelihood of evolutionary branching is influenced by the magnitude of the evolutionary lag. Both of them can also influence the development of food-webs, which might cancel the effect expected above. In this sense, the E-IFD is no more than a candidate for what might be related to the development of complex food-webs.

\section{Numerical simulation}

\section{Calculation methods}

We calculated the evolutionary dynamics based on Eq. 8 using two different methods. The first assumes asexual

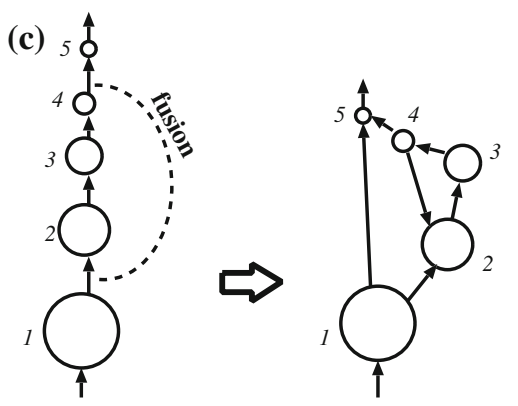

Phenotype space
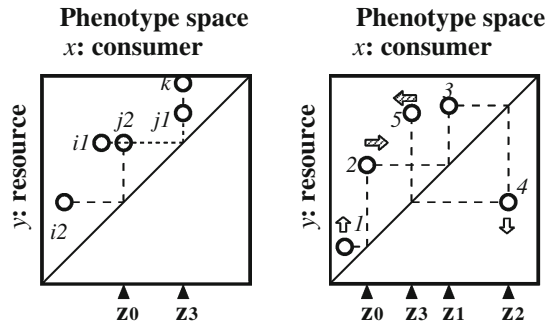

Phenotype space $x$ : consumer
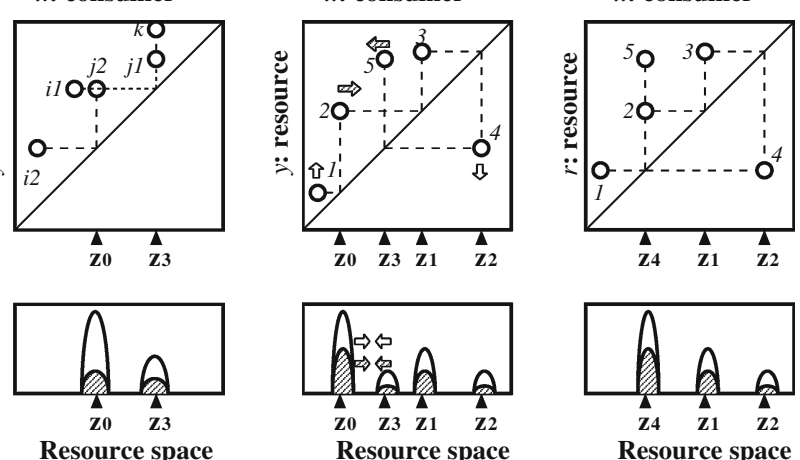

emergence of omnivores and a loop, respectively. The states in the phenotype space and in the resource space are represented in a similar manner to Fig. 2

reproduction. The population dynamics are calculated by discretizing Eq. 8 and mutants slightly deviated from their parent phenotypes are generated at each time step as stochastic events. The basal resource was assumed to be constant by assuming large $v$ (allowing its dynamics to not change the results qualitatively). The consumption-effort and resource patterns were specifically defined as Gaussian distributions. To examine the robustness of our analytical predictions, we assumed that the widths of these patterns are different from each other. Non zero values were also used for $\alpha$ at Eq. 4 for the same purpose.

The second method assumes sexual reproduction, large mutation rates, and very small mutation magnitudes. The effect of mutation is expressed as a deterministic diffusion process of biomasses in the phenotype space, while the effect of mating is expressed by generating an intermediate phenotype from each mating of two phenotypes. For fast calculation, significantly narrow widths were assumed for both the consumption-effort and resource patterns. We call the first and second methods the "asexual-stochastic method" and the "sexual-deterministic method," respectively. See S5 in the ESM for details of the methods.

During the evolutionary dynamics, basically, each phenotypic cluster isolated from the others in the phenotype space was treated as a species. The number of species was counted as the number of "islands" of positive biomass in the phenotype space. When an island has more than one 
biomass peak, each peak was counted as a species in the sexual-deterministic method, in order to detect evolutionary branching in its early stage. The trophic level of each species was calculated based on the amount of basal resource required for the production of unit biomass of the species (Pauly and Christensen 1995) (see S6 in the ESM).

\section{Simulated evolutionary dynamics}

Figure 5 shows typical evolutionary dynamics of the coupled $r$ - and $c$-diversifications in the asexual-stochastic method. Similar widths for the resource and consumptioneffort patterns are assumed in this case. Starting from a single species consuming the basal resource, $c$-diversification occurs and $C(z)$ becomes similar to $R(z)$ in the resource space (Fig. 5d), which makes the evolutionary lag $L$ small (Fig. 5a). Then, $r$-diversification occurs, which induces $c$-diversification of the predator of the diversified prey (Fig. 5e, f). In these processes, the system repeatedly converges to and deviates from E-IFD (Fig. 5a, in which convergence to E-IFD is indicated with horizontal dashed lines). The convergence is realized by convergence of the community to no-lag communities, which can be seen by plotting historical traces of species' $x$ and $y$ (with the colours black and gray, respectively), as shown in Fig. 5b. Although some black lines, corresponding to small values of $x$, are always away from gray lines, they consume the basal resource $I(z)$ that exists there.

Larger resource spaces, in terms of the widths of the consumption-effort and resource patterns, allow diverse species to coexist, as shown in Fig. 6. The evolutionary lag $L$ is kept small in these dynamics (the black curve in Fig. 6a), and a complex food-web develops (Fig. 6c-e). The food-web is maintained through continuous turnover with repeated diversification and extinction. Most of the $r$ diversification occurs as trait substitution rather than evolutionary branching. (This can be because the difference of the two phenotypes in $y$ causes a difference in their competitive ability as prey, but it cannot be compensated through their population dynamics if their $x$ share the same value.) Since the smallness of the evolutionary lag keeps the community close to no-lag communities, evolutionary trees of $x$ (red tree) and $y$ (blue tree) are almost overlapped, as shown in Fig. 6b, except for several red branches at the left side that correspond to the consumption of the basal resources. These red and blue trees are projections of a single evolutionary tree in a three-dimensional space composed of the phenotype space and season axis, like Fig. 7d.

The relationship between small evolutionary lag and food-web development is also observed when the time averages of the numbers of species and links are plotted against the time average of $L$ at different values for the mutation rates $\left(\mu_{x}, \mu_{y}\right)$ or the amount of consumption effort (a)

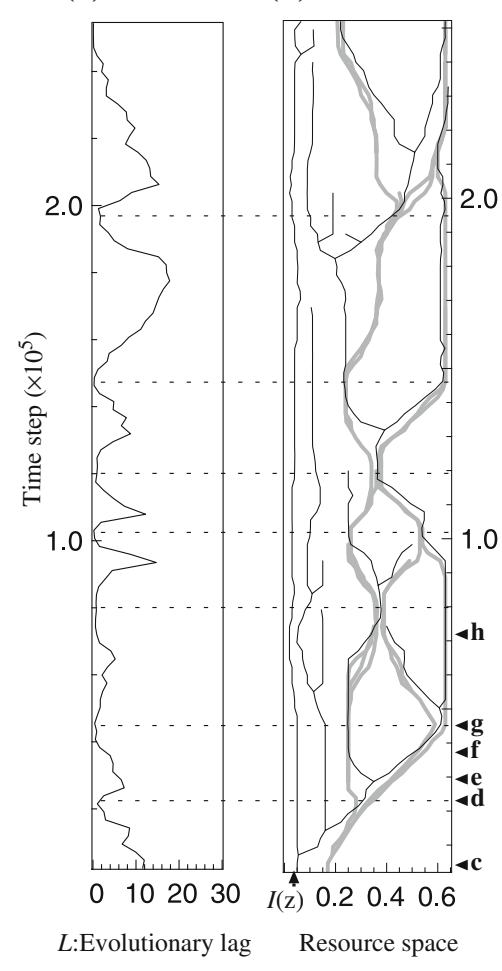

(h)

(g)

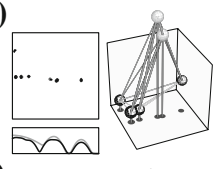

(.

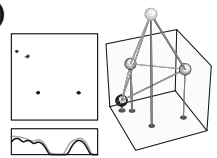

(f)

(e)
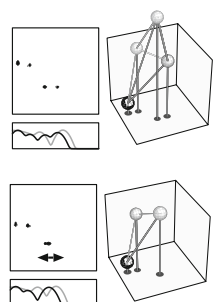

(d)

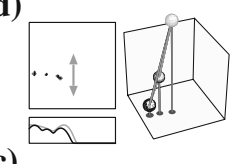

(c)

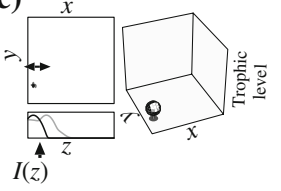

Fig. 5 Repeated evolutionary diversification in simulation with the asexual-stochastic method. The evolutionary lag index $L$ (a), evolutionary traces of $x$ (black curves) and $y$ (gray curves) of each species (b), and snap shots of phenotype distributions and corresponding food-webs $(\mathbf{c}-\mathbf{h})$ are plotted. In each of $\mathbf{c}-\mathbf{h}$, the phenotype distribution (upper left), the total resource and consumption-effort distributions (lower left), and the food-web structure (right) are represented. In the phenotype space, the darker color indicates higher biomass density. The gray vertical and black horizontal arrows indicate the directions of $r$ - and $c$-diversifications, respectively. In the resource space, the resource and consumption-effort distributions are indicated with gray and black curves, respectively, on a logarithmic scale [i.e., $\ln \left(\varepsilon_{L}+R(z)\right)$ and $\ln \left(\varepsilon_{L}+C(z)\right)$ ]. Food-webs are represented in three-dimensional spaces, in which the $x-y$ planes are phenotype spaces and the $z$-axes are trophic levels. Each species is represented as a sphere with its size as the biomass. Species at higher trophic levels are also indicated with lighter colors. The dark and light gray pipes connecting species indicate upward and downward biomass flows among trophic levels, respectively. The pipes from the bottom indicate biomass flows from the basal resource. Model parameters: $\Omega=0.25, \lambda=0.25, d=1, M=400, \alpha=1, \beta=2.5$ $\times 10^{-5}, \gamma=0.01, I(z)=8,000 \times \exp \left(-z^{2} /\left[2 \times 0.08^{2}\right]\right), \varepsilon_{d}=1$ $\times 10^{-5}, \sigma_{c}=0.025, \sigma_{r}=0.024, \mu_{x}=\mu_{y}=1 \times 10^{-9}, \sigma_{\mu}=0.5, x=[0$, 0.7], $y=[0,0.65], \Delta t=0.003, \varepsilon_{L}=1$

$M$. As shown in Fig. 8a, b, the numbers of species and links both tend to have their maximum values when $L$ is small among its observed values, where the number of links per species (i.e., the number of prey per species) also has its maximum. However, the numbers of species and links drop when $L$ is very small. In this case, the evolution of $x$ seems to be relatively too fast to allow for the smooth $r$-diversification. 
Fig. 6 Food-web development and maintenance in simulations with the asexual-deterministic method. The index for evolutionary lag $L(\mathbf{a})$, evolutionary traces of $x$ (red curves) and $y$ (blue curves) of species (b), and their corresponding food-webs $(\mathbf{c}-\mathbf{e})$ are plotted as in the same manner as with Fig. 5. Upward and downward biomass flows are indicated with yellow and green pipes, respectively. In a, the dynamics of $L$ and the numbers of species and links are plotted with black, blue, and red curves, respectively. Model parameters: $M=1, \alpha=1$, $\beta=5.0 \times 10^{-4}, \gamma=0.01$, $I(z)=8,000 \times \exp (-[z$ $\left.-0.18]^{2} /\left[2 \times 0.04^{2}\right]\right), \sigma_{c}=$ $0.015, \sigma_{r}=0.016, \mu_{x}=5$ $\times 10^{-8}, \mu_{y}=1 \times 10^{-7}, y=[0$, $0.95]$; the others have the same values as in Fig. 5 (a)

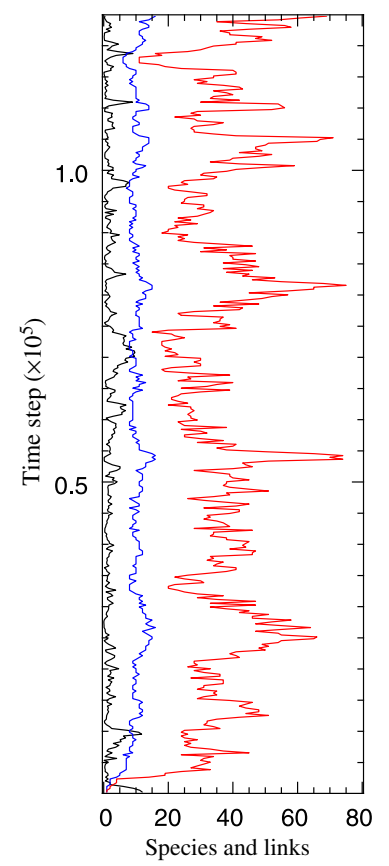

(b)

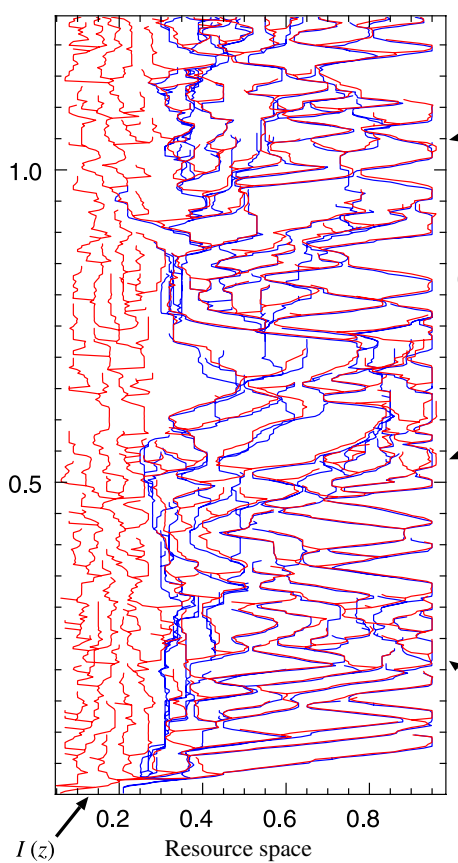

(e)

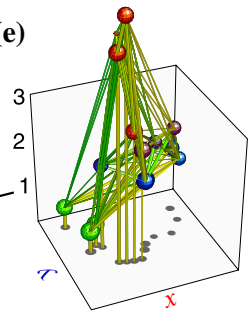

(d)

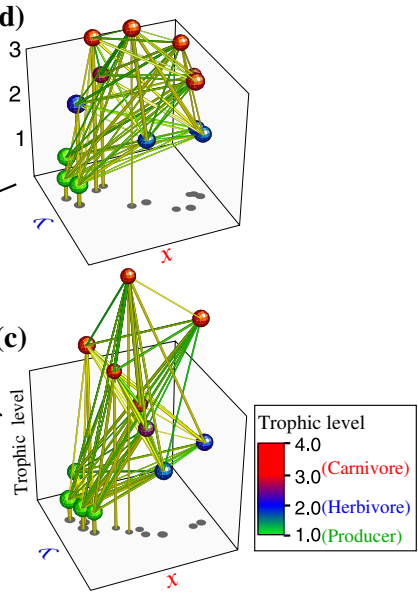

(c)

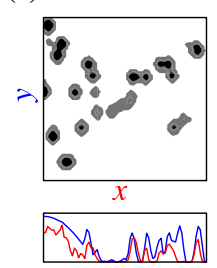

(b)

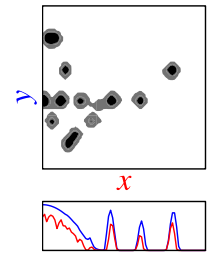

(a)

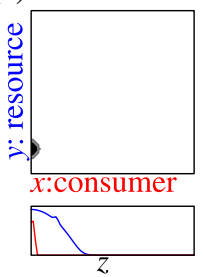

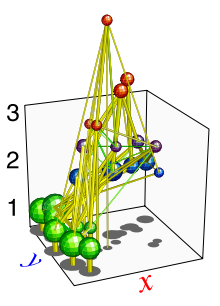

(d)

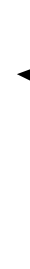

(e)
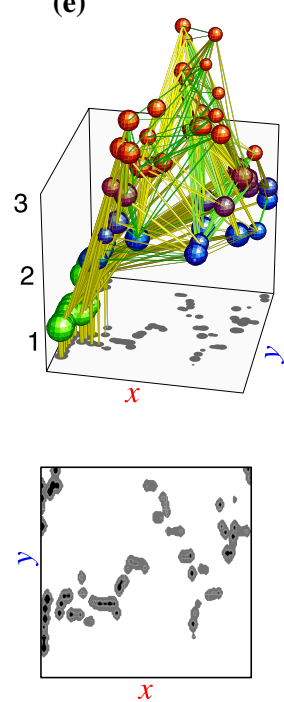

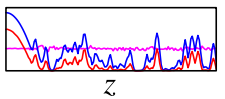

Fig. 7 Food-web development in simulations with the sexual-deterministic method. Each of a-c shows the state at the corresponding time step in d. d is the visualization of the dynamics as an evolutionary tree. $\mathbf{e}$ is a sample of large food-webs maintained for a long period. Food-webs in these panels are plotted in the same manner as with $\mathbf{c}-\mathbf{e}$ in Fig. 6. Phenotype and resource spaces are plotted in the same manner as with $\mathbf{c}-\mathbf{h}$ in Fig. 5, except that the total consumption effort and resource distributions are plotted with red and blue curves, respectively. In the evolutionary tree (d), evolutionary traces of species in the phenotype space are depicted. The width of the pipes indicate the biomasses. Model parameters for a-d: $M=11.0, \Omega=1.8$ $\times 10^{3}, \lambda=0.15, d=1.0, \alpha=0, b=1 \times 10^{2}, \gamma=0.056, D_{x}=4.8$ $\times 10^{-4}, D_{y}=2.0 \times 10^{-3}, \varepsilon_{d}=1.0 \times 10^{-5}, \sigma_{m}=2.8 \times 10^{-2}, \zeta=1.0$, $x=[0,1], y=[0,1], \Delta t=1.0 \times 10^{-2}, L(z)=4,000 \times \exp \left(-z^{2} /\right.$ $\left.\left[2 \times 0.08^{2}\right]\right)$. Model parameters for e: $x=[0,2], y=[0,2], M=10.5$, $\Omega=2 \times 10^{3}, D_{x}=3.6 \times 10^{-4}, D_{y}=2.0 \times 10^{-3}$; the others have the same values as in $\mathbf{d}$ 
If the consumption-effort and resource patterns have quite different widths from each other, historical traces of $x$ and $y$ are not similar to each other. However, it is still observed that food-web development is easy when $L$ is small (Fig. 8c).

Numerical simulation with the sexual-deterministic method also showed food-web development through the coupled $r$ - and $c$-diversifications (Fig. 7). It is also observed that the food-web development is easy when $L$ is small but not too small (Fig. 9).

These numerical results above coincide with the two analytical predictions: (1) the coupled diversifications of $y$ and $x$ may occur when predators have caught up with their prey, which may be repeated; and (2) complex food-webs might develop easily when the evolutionary lag is kept small but not too small.

\section{Discussion}

Analytical understanding of the evolution of complex food-webs

In previous theoretical studies, the evolutionary development of food-webs have been analyzed mainly by numerical simulation (Caldarelli et al. 1998; Yoshida 2003; Drossel et al. 2004; Loeuille and Loreau 2005; Ito and Ikegami 2006). Analytical insights into such dynamics are important for understanding the mechanisms and constraints underlying food-web development. As for the evolutionary dynamics of complex food-webs, such insight is provided only by Matsuda and Namba (1991) as a condition for coevolutionary stable communities (CSC). Here, we provided a new analytical insight as potential evolutionary paths for the development of complex foodwebs, which is a repetition of divisions (i.e., the coupled $r$ and $c$-diversifications) and fusions of interaction groups, keeping the evolutionary lag between predators and prey small. The analytical results are applicable to any food-web structures, and these were supported by the present numerical simulation. Although our results depend on the specific model structures that we assumed, these can be stepping stones for understanding the general features of food-web development. For example, in no-lag communities, the evolutionary stability of $x$ coincides with the condition for CSC. Since CSC does not consider the evolution of prey, the evolution of prey may influence the stability of CSC, as no-lag communities in our model may not be evolutionary-stable because of the evolution of $y$. In addition, since the logic of the coupled $r$ - and $c$-diversifications seems simple and has some generality, similar dynamics may be observed in other evolutionary food-web models (Yoshida 2003; Drossel et al. 2004; Loeuille and Loreau 2005).

The most important finding in our results is the relationship between the evolutionary lag and the development of complex food-webs. We analytically predicted this relationship by expecting that small evolutionary lag enhances the maintenance of complex food-web structures. Although we did not examine the stability of population dynamical equilibria of the states that are close to no-lag communities, the present numerical simulation showed that many of such states, which correspond to complex foodwebs, have stable equilibria that allow the coexistence of their component species. It is notable that the logic of the prediction is, essentially, identical to the general concept of the limiting similarity (MacArthur 1972). In addition, nolag communities correspond to the IFDs (Fretwell and Lucas 1970) in the evolutionary sense. Thus, the existence of the predicted effect might also have some model-independent generality. However, it is notable that the same relationship might be generated without the predicted effect, if the likelihood of evolutionary branching becomes frequent when the evolutionary lag is kept small. At least our finding seems worthy of being studied further in order to make the mechanism clear.

\section{Functional response}

The functional response used in our model, Eq. 4, may correspond to extremely heterogeneous environments in space and/or time (e.g., within a day or year), in which each patch or each season has only a single kind of resource (see $\mathrm{S} 1$ in the ESM). An opposite assumption is completely uniform environments, in which the densities of all kinds of resources do not change along space and season. In this case, a different form of the functional response can be derived:

$g(\mathbf{z}, \mathbf{s})=\frac{M \cdot c(\mathbf{z}, \mathbf{s}) \cdot R(\mathbf{z})}{\alpha+\int c(\tilde{\mathbf{z}}, \mathbf{s}) \cdot[\beta C(\tilde{\mathbf{z}})+\gamma R(\tilde{\mathbf{z}})] \mathrm{d} \tilde{\mathbf{z}}}$

See $\mathrm{S} 1$ in the ESM for its derivation. In this case, $g\left(\mathbf{s}_{j}, \mathbf{s}_{i}\right)$ can be expressed without $z$ :

$g\left(\mathbf{s}_{j}, \mathbf{s}_{i}\right)=\frac{\Omega M \cdot \psi\left(\mathbf{s}_{j}, \mathbf{s}_{i}\right) n_{j}}{\alpha+\beta M \sum_{k} \varphi\left(\mathbf{s}_{k}, \mathbf{s}_{i}\right) n_{k}+\gamma \Omega \sum_{k} \psi\left(\mathbf{s}_{k}, \mathbf{s}_{i}\right) n_{k}}$

where $\varphi\left(\mathbf{s}_{j}, \mathbf{s}_{i}\right)=\int c\left(\mathbf{z}, \mathbf{s}_{i}\right) c\left(\mathbf{z}, \mathbf{s}_{j}\right) \mathrm{d} \mathbf{z}$ is the overlap between $c\left(\mathbf{z}, \mathbf{s}_{i}\right)$ and $c\left(\mathbf{z}, \mathbf{s}_{j}\right)$, while $\psi\left(\mathbf{s}_{j}, \mathbf{s}_{i}\right)=\int c\left(\mathbf{z}, \mathbf{s}_{i}\right) r\left(\mathbf{z}, \mathbf{s}_{j}\right) \mathrm{d} \mathbf{z}$ is the overlap between $c\left(\mathbf{z}, \mathbf{s}_{i}\right)$ and $r\left(\mathbf{z}, \mathbf{s}_{j}\right)$. Equation 27 is the same with the multispecies Beddington-DeAngelis type response proposed by Arditi and Michalski (1996), which is used in an evolutionary food-web model by Drossel et al. (2004). With this response instead of Eq. 4, our model still provides population dynamical equilibria at $C(\mathbf{z})=\hat{A} R(\mathbf{z})$ for no-lag communities under $\alpha=0$ (i.e., ratio-dependent 

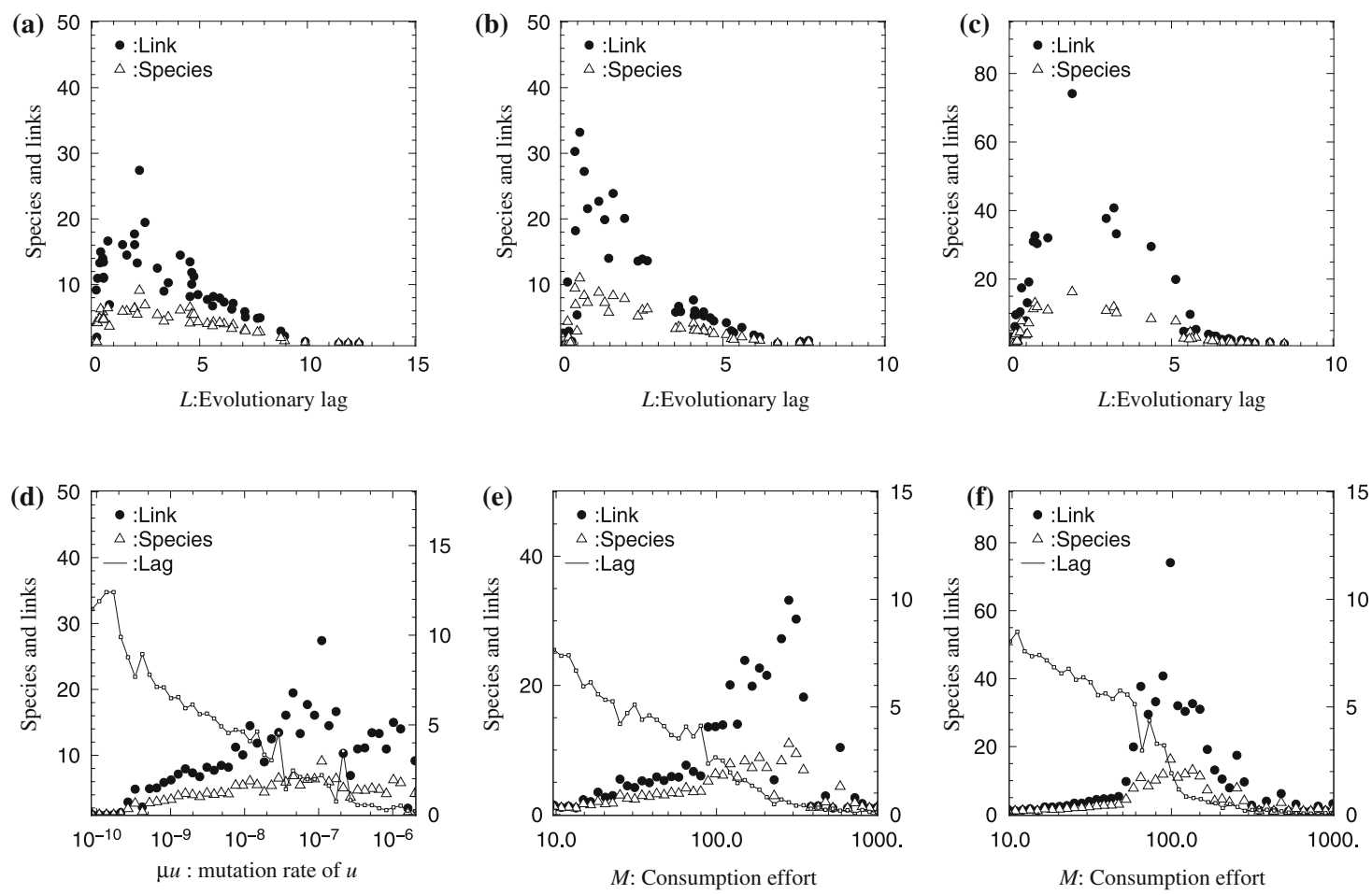

Fig. 8 Relationships between the time averages of evolutionary lag $L$ and those of the numbers of species or links at different values of model parameters. The time-averaged values of the numbers of species and links, and $L$ (against the right axis) are plotted at different values for $\mu_{x}(\mathbf{d})$ or $M(\mathbf{e}$ and $\mathbf{f})$. In the simulation of $\mathbf{f}$, the width of the
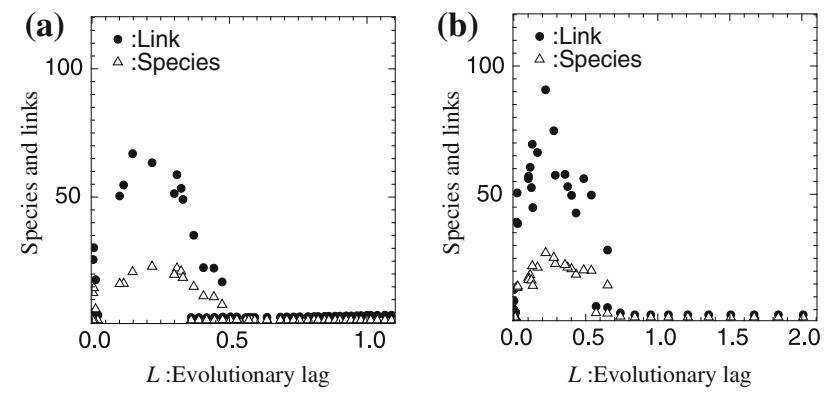

Fig. 9 Relationships between the time averages of the evolutionary lag index $L$ and those of the numbers of species or links at different mutation rates. $\mathbf{a}$ and $\mathbf{b}$ The relationship under various rates for $x$ (from $5 \times 10^{-3}$ to $1 \times 10^{-5}$ ) and $y$ (from $3 \times 10^{-2}$ to $5 \times 10^{-5}$ ), respectively. Model parameters: $M=8, \Delta t=2.0 \times 10^{-2}$; the others have the same values as in Fig. 7e

response), as long as the interaction groups are isolated from each other. Thus, the smallness of the evolutionary lag also seems to be important for food-web development in this case (H. C. Ito, in preparation).

The ratio-dependent response may be expected if prey can move into and out of refuges, and if predators easily find prey that are outside of refuges (Abrams and Ginzburg 2000). Since predators at good locations can find resources easily in this case, it is expected that most of the energy for resource pattern is twice that of the consumption-effort pattern, while these are similar in $\mathbf{d}$ and $\mathbf{e}$. a-c were plotted with the same data as with $\mathbf{d}-\mathbf{f}$, respectively. The time range for averaging is from 3,000 to 100,000 time steps. The fixed model parameters have the same values as in Fig. 5, except for $\sigma_{c}=0.01, \sigma_{r}=0.02$ in $\mathbf{c}$ and $\mathbf{f}$

foraging is consumed for the handling of resources and interference competition. This corresponds to $\alpha \ll \beta U(z)$ $+\gamma R(z)$ in $\phi(z)$. Then, $\phi(z)=1 /[\alpha+\beta U(z)+\gamma R(z)] \simeq 1 /$ $[\beta U(z)+\gamma R(z)]$ approximately gives a ratio-dependent response. However, functional responses have large variations in real populations (Abrams and Ginzburg 2000). Drossel et al. (2004) numerically showed that the types of functional response influences the complexity of evolutionarily formed food-web structures. Thus, further investigation assuming various functional responses is needed in order to examine the robustness of our presented scenario for evolutionary food-web development.

\section{Coevolutionary diversification}

Ito and Ikegami (2006) has shown that food-webs develop with repeated $r$ - and $c$-diversifications by numerical simulation, but when such diversifications occur was not clear. In addition, Ito and Ikegami (2006) assumed that both the consumption-effort and resource patterns are significantly narrow (i.e., the delta function), and that both population dynamics and mutation are deterministic. Both of these assumptions may not be realistic. In our present study, we assumed certain widths for the consumption-effort and resource patterns. Under this assumption, we analytically 
showed that $r$-diversification may occur when the predators have caught up with their prey, and that the $r$-diversification may induce $c$-diversification. This was confirmed by numerical simulation assuming stochasticity in the population dynamics and mutation (i.e., the asexual stochastic method). Although an identical shape for consumptioneffort and resource patterns is assumed to obtain analytical results, the numerical simulation showed successful foodweb developments when the evolutionary lag is kept small, even if the resource pattern is twice as wide as the consumption-effort pattern, as shown in Fig. 8c. The dynamics seems robust to some extent, as shown in Figs. 8 and 9 .

In the present analyses, we focused on the total consumption-effort distribution $C(z)$ and the total resource distributions $R(z)$ in the resource space, instead of $x, y$ and the biomasses of species. From this perspective in the resource space, an arbitrary community with any complex food-web can be treated as if it has only two trophic levels, $C(z)$ and $R(z)$. Thus, the conducted analyses on directional and diversifying selections seem to essentially be the same as with previous theoretical studies on predator-prey coevolution with two fixed trophic levels (Brown and Vincent 1992; Dieckmann and Law 1996; Abrams and Matsuda 1997; Abrams 2000; Doebeli and Dieckmann 2000; Kisdi 2006). Indeed, the logic of the coupled $r$ - and $c$-diversification seems the same as with Brown and Vincent (1992) and Doebeli and Dieckmann (2000). Moreover, those studies have analytically and numerically shown that various population dynamical and evolutionary outcomes, including not only the coevolutionary diversification of predator and prey (Doebeli and Dieckmann 2000; Kisdi 2006) and extinction (Dieckmann and Law 1996), but also their population cycle (Abrams and Matsuda 1997), evolutionary cycles without diversification (Dieckmann and Law 1996; Abrams and Matsuda 1997) are possible, depending on the ecological and mutational settings. In our model, the coupled diversification becomes difficult when the consumption-effort pattern is sufficiently wider than that of the resource pattern, which coincides with Brown and Vincent (1992). In addition, the population cycle and evolutionary cycle are also observed in numerical simulations occasionally, although the conditions for them have not been investigated. Further analytical and numerical studies are needed to clarify the possible variety of evolutionary dynamics in our model.

However, it is not easy to analyze multispecies coevolution in two-dimensional phenotype (or trait) space. In the case of one-dimensional trait space, formal conditions for evolutionary branching are following; the condition for convergence stability (existence of evolutionary attractor $s^{*}$ at which directional selection vanishes) (Abrams et al. 1993), the condition for evolutionary instability (disruptive selection at $s^{*}$ ) (Maynard Smith 1982), and the condition for mutual invasibility (coexistence of two slightly diverged phenotypes in the neighborhood of $s^{*}$ ) (MacArthur 1972). Evolutionary branching is assured if all of the three conditions are fulfilled (Geritz et al. 1997).

In the case of multispecies coevolution in multidimensional trait spaces, formal conditions for evolutionary branching have not yet been obtained. But for convergence stability, the conditions have been obtained (Leimar 2008). In addition, obtaining conditions for evolutionary instability does not seem to be difficult (Ackermann and Doebeli 2004; Egas et al. 2005; Leimar 2005; Ito and Shimada 2007). If there is a convergence stable state that is evolutionarily unstable, the prediction of evolutionary diversification is not difficult (Ackermann and Doebeli 2004; Egas et al. 2005; Ito and Shimada 2007).

In our present model, however, some of the evolutionary diversifications important for food-web development occur in the absence of a convergence stable state (not shown). In this case, our obtained analytical conditions still indicates the possibility of evolutionary diversification, but they cannot assure its likelihood. The existence of such evolutionary branching is reported by Leimar (2005) and Ito and Dieckmann (2007). According to Ito and Dieckmann (2007), if species $i$ experiences disruptive selection in $x_{i}$, evolutionary branching in $x_{i}$ is expected when the evolution of $r_{i}$ is sufficiently slow (and vice versa). By examining directional selections and diversifying/stabilizing selections simultaneously, the likelihood of such evolutionary branching can be provided (H. C. Ito and U. Dieckmann, in preparation). In addition, Ito and Dieckmann (2007) showed turnover dynamics with repeated diversification similar to Fig. $6 \mathrm{~b}$ by the combination of disruptive selection in one trait and directional selection in another trait. Although either of which $x$ and $y$ experiences disruptive (or directional) selection can change in our model, the basic mechanism seems the same.

Uniformity of the competitive strength for food-web development

In our model, similarity in competitive strengths among species seems to be important for the development of complex food-webs. Such similarity among species can be translated into a uniformity of competitive strengths in the community. Here, we discuss this under slightly more generalized assumptions. Since sufficiently small $\alpha$ leads to the same tendency with the case of $\alpha=0$, we assume $\alpha=0$ in the following.

Our model assumes that all species are identical in their trophic efficiency $\lambda$, the amount of consumption effort $M$, vulnerability $\Omega$, and natural death rate $\rho$. Here, we consider a case that species can have different values for these 
parameters compared to each other. In this case, $C(z)$ and $R(z)$ are given by $\sum_{i} M_{i} n_{i} c\left(z, \mathbf{s}_{i}\right)$ and $\sum_{i} \Omega_{i} n_{i} r\left(z, \mathbf{s}_{i}\right)$, respectively. According to Eq. $8, C(z)=\hat{A R}(z)$ still provides population dynamical equilibrium and identical competition strength, if the ratio $\lambda_{i} M_{i}: \Omega_{i}: \rho_{i}$ is kept identical among those species. Thus, if the variation of the ratio among them is kept small, complex food-webs may develop through evolutionary dynamics with small evolutionary lag. Conversely, if the variation of the ratio is large, even a no-lag community has a large variation in competitive strength among species. In this case, species with large $\lambda M$ and with small $\Omega$ and $\rho$ are strong competitors, according to Eq. 8. The strong species can exclude the other weaker ones at each fusion of the interaction groups. Such an exclusion is likely to be accompanied by evolutionary diversification of the strong species, generating their daughter species (Ito and Dieckmann 2007). Repetition of the fusion results in a community composed of only the strong daughter species, having similar values for the ratio to each other. Then, they may form a complex foodweb.

However, the parameters $\lambda, M, \Omega$, and $\rho$ may evolve simultaneously with $x$ and $y$. In this case, the variation of the ratio among the daughter species can arise through their independent evolution (see also Ito and Dieckmann 2007). When the growth rate of the variation is fast compared to the frequency of the fusion, the variation may be kept large. This seems to make food-web development difficult. Thus, the development of a complex food-web requires that the evolution of the ratio becomes slow, so that the variation of the ratio grows slowly compared to the evolutionary speed of $x$ and $y$. In this case, the division and fusion of interaction groups may be repeated before the variation becomes large. Then, complex food-webs may develop.

Even after a complex food-web has developed, the parameters may still evolve in the directions that keep the ratio $\lambda M: \Omega: \rho$ similar. Such directionality of evolutionary changes can be understood as a trade-off function. Large $\lambda M$ gives large biomass production, small $\Omega$ and $\rho$ give small biomass loss by predation and by energy consumption, respectively. But the larger production has to be accompanied by the larger predation risk and the larger consumption rate in order to maintain the ratio. In this case, various species with different parameter values may coexist.

In the above discussion, the energy for the handling resource, $\gamma$, is still assumed to be identical among resources. Variation of the handling resource can be introduced by assuming $\gamma$ as a function of $z \cdot \gamma$ can also be a evolutionary trait for prey, such as the thickness of a shell. Under such an assumption, however, $C(z)=\hat{A} R(z)$ and $\alpha=0$ may not provide identical competitive strength among species. In this case, further generalization of the concept of evolutionary lag might be required in order to obtain a good condition for the uniformity.

Comparison with empirical data

Since we are concerned with a minimal logic to explain the evolutionary development of food-webs as our first step, several ecological functions or properties, including functional response, trophic efficiency, and the energy consumption for handling resources, were assumed to be identical among phenotypes or among resources. Those assumptions are not realistic. Nevertheless, some of our assumptions and results might be worthy of comparison with real populations. Below, we qualitatively compare our main assumptions and results with empirical data.

First, we consider how the relationship between $x$ and $y$ (or $c(z, x)$ and $r(z, y)$ ) can be translated into traits in real predator-prey interaction. Possible combinations of predator-prey strategies are predator's jaw (or claw) and prey's shell, prey's toxin and predator's antitoxin, or predator's eye, prey's protective coloration, etc. (see also Abrams 2000). As for the first and second cases, strong evidence for the coevolution of the predators' and prey's traits come from bills of crossbills and corns of hemlock (Parchman and Benkman 2002), and from garter snakes and newts (Geffeney et al. 2005). The claws of crabs and the shells of mollusks are another example of the first case. In this case, mollusks shows evolutionary diversification in their shell morphologies, which seems to be protection from the claws of crabs (Vermeij 1987). Although clear evolutionary response of the claws of crabs to the shell evolution has not reported, the claws of shame-faced crabs (genus Calappa) may be an example. The unique shapes of their claws are specialized to hold and break the thick shells of snails, which seem meaningless in the absence of the snails with these shells.

In our model, $M$ may correspond to the size of the bill (or claw) or the production amount of antitoxin, while $\Omega$ and $h$ may correspond to the thickness of the shell or the production amount of toxin. The $x$ and $y($ or $c(z, \mathbf{s})$ and $r(z, \mathbf{s}))$ correspond to the other possible changes of them that influence the rate of predator-prey interaction, keeping the total energetic cost the same. For example, $y$ may correspond to the shape of the shell, the location of spikes on it, or the location of the prey's body that excretes toxin, while $x$ may correspond to the shape of the bill, the dentition pattern of a claw, or the method of handling so as not to suffer from the prey's toxin.

As for the coupled $r$ - and $c$-coevolution observed in our model, which is our first result, it may correspond to the coevolutionary diversifications of bills of crossbills and corns of hemlock (Parchman and Benkman 2002), or those of garter snakes and newts (Geffeney et al. 2005). In both 
cases, however, the thickness of corn scales and the size of bills, and the strengths of toxin and antitoxin are the detected evolutionary traits in Parchman and Benkman (2002) and Geffeney et al. (2005), respectively. These seem to correspond to $M, \Omega$, and $h$, rather than $x$ and $y$. Another discrepancy is that most of the reported evolutionary diversifications are thought to be parapatric or allopatric, including Parchman and Benkman (2002) and Geffeney et al. (2005), while our model does not have a spatial axis (i.e., sympatric setting). This discrepancy may be reduced if a spatial axis is introduced to the resource space in our model, such that the resource distribution has an environmental gradient. This is because Doebeli and Dieckmann (2003) numerically demonstrated that an ecological interaction favoring sympatric diversification easily induces parapatric speciation under environmental gradients.

Our second result is that the development of complex food-webs may be easy if the functional response is close to the ratio-dependent response and if the deviation from EIFD is kept small. The result was obtained by assuming identical energy consumption for handling resources. The small variation of the ratio $\lambda M: \Omega: \rho$ among species is an additional condition for the case that these parameters also evolve as properties of species. The two conditions regarding E-IFD and the ratio $\lambda M: \Omega: \rho$, respectively, may be examined if we focus on local food-webs in which the variation of energy consumption for handling is small and the functional response is close to the ratio-dependent response. However, ecological data in paleontological records are scarce (but see Crame 1990), and we could not find suitable data for the examination. Although there are studies that report IFD in present populations (Begon et al. 1996), whether IFD is a general phenomenon at the community level is not clear.

By translating the second result into features of evolutionary dynamics, a weak prediction can be made as follows. The two conditions are for realizing the uniformity of competitive strength, which corresponds to an almost flat fitness landscape around existing phenotypes in the phenotype space. In this situation, evolutionary dynamics is expected to be slow. Then, successful food-web development and maintenance may arise from slow evolutionary diversifications and turnovers, respectively. Conversely, rapid evolutionary diversification may correspond to the emergence of large variations of competitive strength, which can cause mass extinction through rapid exclusion by the diversifying lineage. Thus, it may be predicted that rapid evolutionary diversification tends to be accompanied by mass extinction. A widespread view is that external factors are the main causes of mass extinction detected in fossil records (Morris 1998), in which rapid radiation is expected after the extinctions. However, there are empirical studies that support the simultaneous occurrence of the radiation of mammals and the mass extinctions of dinosaurs (Springer et al. 2003). Moreover, they might have been interacting strongly, because fossil record shows that large mesozoic mammals fed on young dinosaurs ( $\mathrm{Hu}$ et al. 2005).

Finally, our second result also implies that every species should be consumed by others (predators and/or parasites). This seems to hold, since we do not know of any species that is completely free from exploitation in the present biological communities forming complex food-webs.

Acknowledgments The authors thank Hiroyuki Matsuda, Ulf Dieckmann, the editor, and the anonymous reviewer for their valuable comments on earlier versions of this manuscript. H.C.I. also thanks David Munro for inventing a tool for numerical analysis and visualization with great performance and flexibility, named Yorick, and distributing it for free. All of the figures in this study were produced with Yorick. H.C.I. acknowledges support in the form of a Research Fellowship for Young Scientists by the Japan Society for the Promotion of Science (JSPS).

\section{References}

Abrams PA (2000) The evolution of predator-prey interactions: theory and evidence. Annu Rev Ecol Syst 31:79-105. doi: 10.1146/annurev.ecolsys.31.1.79

Abrams PA, Ginzburg LR (2000) The nature of predation: prey dependent, ratio dependent or neither? Trends Ecol Evol 15:337341. doi:10.1016/S0169-5347(00)01908-X

Abrams PA, Matsuda H (1997) Fitness minimization and dynamic instability as a consequence of predator-prey coevolution. Evol Ecol 11:1-20. doi:10.1023/A:1018445517101

Abrams PA, Matsuda H, Harada Y (1993) Evolutionarily unstable fitness maxima and stable fitness minima of continuous traits. Evol Ecol 7:465-487. doi:10.1007/BF01237642

Ackermann M, Doebeli M (2004) Evolution of niche width and adaptive diversification. Evolution 58:2599-2612

Arditi R, Ginzburg LR (1989) Coupling in predator-prey dynamics: ratio-dependence. $\mathrm{J}$ Theor Biol 139:311-326. doi:10.1016/ S0022-5193(89)80211-5

Arditi R, Michalski J (1996) Nonlinear food web models and their responses to increased basal productivity. In: Polis GA, Winemiller KO (eds) Food webs: integration of patterns and dynamics. Chapman and Hall, New York, pp 122-133

Beddington JR (1975) Mutual interference between parasites or predators and its effect on searching efficiency. J Anim Ecol 44:331-340. doi: $10.2307 / 3866$

Begon M, Townsend CR, Harper JL (1996) Ecology: individuals, populations and communities, 3rd edn. Blackwell Science, London

Brown JS, Vincent TL (1992) Organization of predator-prey communities as an evolutionary game. Evolution 46:12691283. doi: $10.2307 / 2409936$

Caldarelli G, Higgs PG, McKane AJ (1998) Modelling coevolution in multispecies communities. J Theor Biol 193:345-358. doi: 10.1006/jtbi.1998.0706

Crame JA (1990) Trophic structure. In: Briggs DEG, Crowther PR (eds) Paleobiology: a synthesis, 1st edn. Blackwell, Oxford, pp 385-391

DeAngelis DL, Goldstein RA, O'Neill RV (1975) A model for tropic interaction. Ecology 56:881-892. doi:10.2307/1936298 
Dercole F (2003) Remarks on branching-extinction evolutionary cycles. J Math Biol 47:569-580. doi:10.1007/s00285-0030236-4

Di Giulio M (2001) The universal ancestor was a thermophile or a hyperthermophile. Gene 281:11-17. doi:10.1016/S0378-1119 (01)00781-8

Dieckmann U, Doebeli M (1999) On the origin of species by sympatric speciation. Nature 400:354-357. doi:10.1038/22521

Dieckmann U, Law R (1996) The dynamical theory of coevolution: a derivation from stochastic ecological processes. J Math Biol 34:579-612. doi:10.1007/BF02409751

Doebeli M, Dieckmann U (2000) Evolutionary branching and sympatric speciation caused by different types of ecological interactions. Am Nat 156:S77-S101. doi:10.1086/303417

Doebeli M, Dieckmann U (2003) Speciation along environmental gradients. Nature 421:259-264. doi:10.1038/nature01274

Drossel B, McKane AJ, Quince C (2004) The impact of nonlinear functional responses on the long-term evolution of food web structure. J Theor Biol 229:539-548. doi:10.1016/j.jtbi.2004. 04.033

Egas M, Sabelis MW, Dieckmann U (2005) Evolution of specialization and ecological character displacement of herbivores along a gradient of plant quality. Evolution 59:507-520

Fretwell SD, Lucas HL Jr (1970) On territorial behavior and other factors influencing habitat distribution in birds. I. Theoretical development. Acta Biotheor 19:16-36. doi:10.1007/ BF01601953

Geffeney SL, Fujimoto E, Brodie ED 3rd, Brodie ED Jr, Ruben PC (2005) Evolutionary diversification of TTX-resistant sodium channels in a predator-prey interaction. Nature 434:759-763. doi:10.1038/nature03444

Geritz SAH, Metz JAJ, Kisdi E, Meszéna G (1997) Dynamics of adaptation and evolutionary branching. Phys Rev Lett 78:20242027. doi:10.1103/PhysRevLett.78.2024

Geritz SAH, Kisdi E, Meszéna G, Metz JAJ (1998) Evolutionarily singular strategies and the adaptive growth and branching of the evolutionary tree. Evol Ecol 12:35-57. doi:10.1023/A: 1006554906681

Holling CS (1959) Some characteristics of simple types of predation and parasitism. Can Entomol 91:385-398

Hu Y, Meng J, Wang Y, Li C (2005) Large Mesozoic mammals fed on young dinosaurs. Nature 433:149-152. doi:10.1038/ nature 03102

Ito HC, Dieckmann U (2007) A new mechanism for recurrent adaptive radiations. Am Nat 170:E96-E111. doi:10.1086/521229

Ito HC, Ikegami T (2006) Food-web formation with recursive evolutionary branching. J Theor Biol 238:1-10. doi:10.1016/ j.jtbi.2005.05.003
Ito HC, Shimada M (2007) Niche expansion: coupled evolutionary branching of niche position and width. Evol Ecol Res 9:675-695

Kisdi E (2006) Trade-off geometries and the adaptive dynamics of two co-evolving species. Evol Ecol Res 8:959-973

Kisdi E, Geritz SAH (1999) Adaptive dynamics in allele space: evolution of genetic polymorphism by small mutations in a heterogeneous environment. Evolution 53:993-1008. doi: $10.2307 / 2640805$

Kisdi E, Geritz SAH (2001) Evolutionary disarmament in interspecific competition. Proc R Soc Lond B Biol Sci 268:2589-2594. doi: $10.1098 / \mathrm{rspb} .2001 .1842$

Leimar O (2005) The evolution of phenotypic polymorphism: randomized strategies versus evolutionary branching. Am Nat 165:669-681. doi:10.1086/429566

Leimar O (2008) Multidimensional convergence stability and the canonical adaptive dynamics. In: Dieckmann U, Metz JAJ (eds) Elements of adaptive dynamics. Cambridge University Press, Cambridge (in press)

Loeuille N, Loreau M (2005) Evolutionary emergence of sizestructured food webs. Proc Natl Acad Sci USA 102:5761-5766. doi:10.1073/pnas.0408424102

MacArthur RA (1972) Geographical ecology: patterns in the distribution of species. Harper and Row, New York

Matsuda H, Namba T (1991) Food web graph of a coevolutionarily stable community. Ecology 72:267-276. doi:10.2307/1938920

Maynard Smith J (1982) Evolution and the theory of games. Cambridge University Press, Cambridge

Metz JAJ, Geritz SAH, Meszéna G, Jacobs FJA, van Heerwaarden JS (1996) Adaptive dynamics: a geometrical study of the consequences of nearly faithful reproduction. In: van Strien SJ, Verduyn Lunel SM (eds) Stochastic and spatial structures of dynamical systems. North Holland, Amsterdam, pp 183-231

Morris SC (1998) The evolution of diversity in ancient ecosystems: a review. Philos Trans R Soc B Biol Sci 353:327-345

Parchman TL, Benkman CW (2002) Diversifying coevolution between crossbills and black spruce on Newfoundland. Evolution 56:1663-1672

Pauly D, Christensen V (1995) Primary production required to sustain global fisheries. Nature 374:255-257. doi:10.1038/374255a0

Springer MS, Murphy WJ, Eizirik E, O’Brien SJ (2003) Placental mammal diversification and the cretaceous-tertiary boundary. Proc Natl Acad Sci USA 100:1056-1061. doi:10.1073/pnas. 0334222100

Vermeij GJ (1987) Evolution and escalation: an ecological history of life. Princeton University Press, Princeton

Yoshida K (2003) Evolutionary dynamics of species diversity in an interaction web system. Ecol Model 163:131-143. doi:10.1016/ S0304-3800(02)00417-9 